\title{
Nonlinearly determined wavefronts of the Nicholson's diffusive equation: when small delays are not harmless
}

\author{
Zuzana Chladnáa ${ }^{\mathrm{a}}$ Karel Hasík ${ }^{\mathrm{b}}$, Jana Kopfováb, Petra Nábělkováb, \\ Sergei Trofimchuk ${ }^{\mathrm{c}, *}$ \\ ${ }^{a}$ Department of Applied Mathematics and Statistics, Faculty of Mathematics, Physics and \\ Informatics, Comenius University, Mlynská dolina, 84248 Bratislava, Slovak Republic \\ ${ }^{b}$ Mathematical Institute, Silesian University, 74601 Opava, Czech Republic \\ ${ }^{c}$ Instituto de Matemática y Física, Universidad de Talca, Casilla 747, Talca, Chile
}

\begin{abstract}
By proving the existence of non-monotone and non-oscillating wavefronts for the Nicholson's blowflies diffusive equation (the NDE), we answer an open question from 16. Surprisingly, these wavefronts can be observed only for sufficiently small delays. Similarly to the pushed fronts, obtained waves are not linearly determined. In contrast, a broader family of eventually monotone wavefronts for the NDE is indeed determined by properties of the spectra of the linearized equations. Our proofs use essentially several specific characteristics of the blowflies birth function (its unimodal form and the negativity of its Schwarz derivative, among others). One of the key auxiliary results of the paper shows that the Mallet-Paret-Cao-Arino theory of super-exponential solutions for scalar equations can be extended for some classes of second order delay differential equations. For the new type of non-monotone waves to the NDE, our numerical simulations also confirm their stability properties established by Mei et al.
\end{abstract}

Keywords: non-linear determinacy, delay, wavefront, existence, super-exponential solution

2010 Mathematics Subject Classification: 34K12, 35K57, 92D25

e-m̌rails addresses: chladna@fmph.uniba.sk (Zuzana Chladná); karel.hasik@math.slu.cz (Karel Hasík); jana.kopfova@math.slu.cz (Jana Kopfová); petra.nabelkova@math.slu.cz (Petra Nábělková); trofimch@inst-mat.utalca.cl (Sergei Trofimchuk, corresponding author) 


\section{Introduction and main results}

Nicholson's blowflies delay differential equation

$$
u^{\prime}(t)=-\delta u(t)+p u(t-\tau) e^{-a u(t-\tau)}, \quad u \geq 0,
$$

was introduced in 1980 by Gurney, Blythe and Nisbet [21] to provide a better description of the evolution of the population $u(t)$ of mature adults of the Australian sheep-blowflies (Lucilia cuprina) observed in a series of highly careful laboratory experiments realized by A. J. Nicholson 33. The positive parameters $\delta, p, a, \tau$ are the model's specific constants and simple scaling of variables allows us to assume that $\delta=a=1$ without any restriction of generality. Equation (1) was introduced as a more elaborate alternative to the delayed logistic equation: in difference with (1), the latter was unable to explain some irregular oscillations of $u(t)$ observed in the collected experimental data. In a short time, it became clear that Nicholson's blowflies equation represents a fascinating and non-trivial object of investigation from the dynamical point of view. This fact attracted the interest of numerous researchers over the decades, cf. 4, 15, 29, 33, 35, 45. Moreover, following the same logic as in the case of the delayed logistic equation (cf. [3]), in 1996 Yang and So [45] introduced the following diffusive version of (1):

$$
\partial_{t} u(t, x)=\partial_{x x} u(t, x)-u(t, x)+p u(t-\tau, x) e^{-u(t-\tau, x)}, \quad x \in \mathbb{R} .
$$

The positive semi-wavefronts $u(t, x)=\phi(x+c t), \phi(-\infty)=0, \liminf _{t \rightarrow+\infty} \phi(t)>0$, are the fundamental transitory regimes in the dynamics generated by the diffusive Nicholson's equation. The existence, uniqueness, oscillation/monotonicity and stability properties of these waves were studied, among many other works, in [7, 12, 15, 16, 26, 27, 37, 38, 39, 44, and the non-local version of (2) was considered, among many other articles, in [17, 18, 19, 20, 24, 25, 28, 36, 46, 47.

In this paper, we revisit the topic of possible shapes for wavefronts $u(t, x)=\phi(x+c t)$, $\phi(-\infty)=0, \phi(+\infty)=\ln p$, of equation (2). Our first main result, Theorem 1 shows that contrarily to the tacitly accepted hypothesis, cf. [7, 27, of the determinacy of the shape of $\phi(t)$ by the spectra of linearized equations at the equilibria (as it happens, for instance, in the delayed or nonlocal KPP-Fisher equations [8, 11]), equation (2) can have wavefronts which are neither monotone nor oscillating even if the linearization of the profile equation at the positive equilibrium has negative eigenvalues. This implies that a non-monotone wave with unusually high leading edge (see Fig. 1) can appear in (2) even if the associated linearized equations predict the existence of exclusively monotone waves. Surprisingly enough, this strange type of wavefront's behavior can occur for arbitrarily small delays $\tau$, to some extent contradicting the folklore principle "Small delays are harmless" of the theory of delay differential equations [35]. The mechanism behind this loss of monotonicity of wavefronts is precisely the same one which causes the "linear determinacy principle" 23 to fail for the monostable population models possessing the weak Allee effect (leading to the appearance of pushed or non-linearly determined waves). In order to state our first result, we introduce some notation. In the sequel, $\Gamma(z, s)$ will denote the lower incomplete gamma function, $\chi(z)$ will denote the characteristic function of equation (1) with $\delta=1$ linearized at $u=0$ :

$$
\Gamma(z, s)=\int_{0}^{z} t^{s-1} e^{-t} d t, \quad \chi(z)=z+1-p e^{-z \tau} .
$$




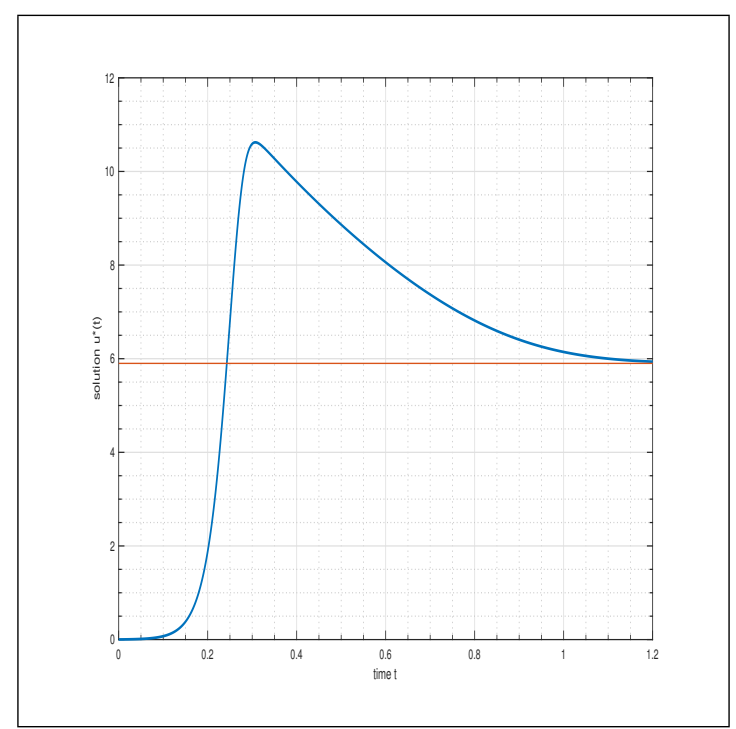

Figure 1: Non-monotone non-oscillating wavefront for equation with $\tau=0.07, p=365$ and $c=+\infty$.

It is easy to see that for each $p>1$ equation $\chi(z)=0$ has exactly one positive root $\mu$. Set $\bar{q}_{2}:=-p e^{-2 \mu \tau} / \chi(2 \mu)<0, m:=\mu^{-1}$ and

$\zeta:=\left(1+\bar{q}_{2}\right) e^{-\tau}+p m\left(\Gamma(1, m+1)-\Gamma\left(e^{-\mu \tau}, m+1\right)+\bar{q}_{2} \Gamma(1, m+2)-\bar{q}_{2} \Gamma\left(e^{-\mu \tau}, m+2\right)\right)$.

Theorem 1. Let $p, \tau$ be such that $p \in \mathfrak{I}:=\left(e^{2}, \exp (1+\exp (-1-\tau) / \tau)\right)$ and $\zeta>\ln p$. Then there exists $\hat{c}(\tau, p)>0$ such that for each $c \geq \hat{c}(\tau, p)$ equation (2) has a positive wavefront propagating with the speed $c$ and whose profile $\phi_{c}(t)$ is eventually monotone at $\pm \infty$ and is non-monotone on $\mathbb{R}$. In fact, $\left|\phi_{c}(\cdot)\right|_{\infty} \geq \zeta>\ln p$.

Example 2. Consider 'small' delay $\tau=0.07$ and take $p=365 \in \mathfrak{I}$. In such a case, formal linear analysis predicts the existence of a unique monotone wavefront connecting 0 and $\ln p$. However, it is easy to find that $\mu=33.64 \ldots, \bar{q}_{2}=-0.05 \ldots$ and $\zeta=6.46 \ldots$ $>\ln p=5.89 \ldots$ Therefore the conclusions of Theorem 1 hold for equation (2) with $\tau=0.07, p=365$. Figure 1 shows a very accurate approximation of the scaled profiles $\phi_{c}(c t)$ of the corresponding non-monotone and non-oscillating wavefronts (nm-waves, for short) in the limit case $c=+\infty$. This picture perfectly agrees with the considerations of Remark 17 where we present a numerical solution of (2) with $\tau=0.07, p=365$ converging to a wavefront propagating with the large but finite speed $c \approx 50$.

Theorem 1 and Example 2 answer in positive an open question raised in [16, p. 53] about the existence of an eventually monotone and non-monotone front of $(2)$ for some $p>e^{2}$. In fact, there are many such wavefronts: on Fig. 2, we present the set of parameters $(\tau, \ln \ln p)$ satisfying all requirements of Theorem 11. Formally, Theorem 1 works for sufficiently fast wavefronts: since the minimal speed of propagation $c_{*}=7.89 \ldots$ in Example 2 is relatively large (in the sense that $c_{*}^{-2}=0.016 \cdots \ll \tau$ ), one might expect 
that even the minimal wavefront in Example 2 has non-monotone and non-oscillating profile. Our numerical simulations confirm this informal conclusion: on Fig. 3, we present three consecutive positions (at the times $t=1,3,5$ ) of solution for equation (2) with $\tau=0.07, p=365$ and with the Heaviside step function as the initial datum. This numerical solution was obtained by applying the second order central difference schemes for the space derivative. The resulting transformed system of ordinary delayed equations has been solved by Matlab built-in function dde23. We take $x \in[-500,500]$ and $t \in[0,5]$. This numerical result complements the discussion in Section 7 of [7].

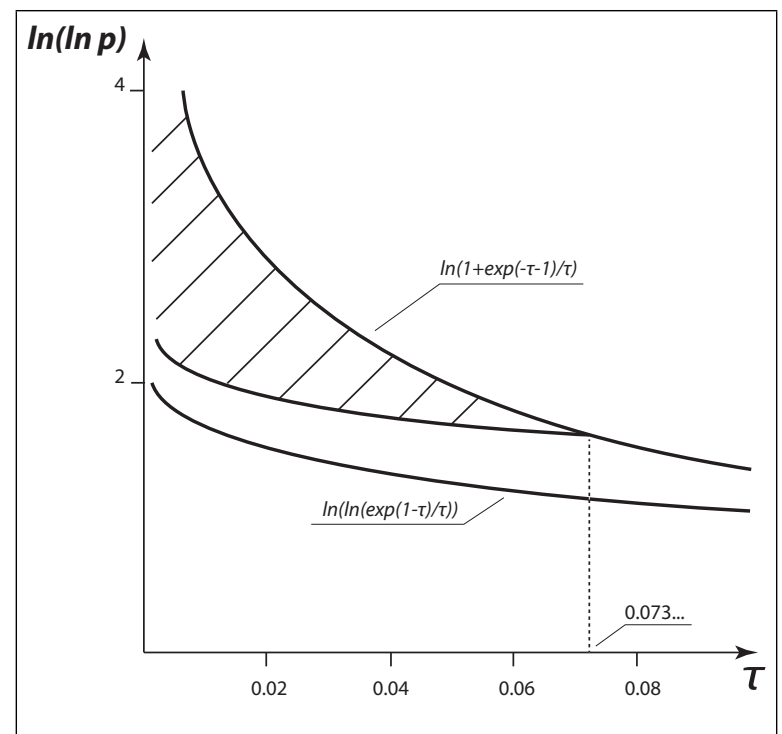

Figure 2: Dashed domain corresponds to parameters $(\tau, p)$ satisfying the assumptions of Theorem 1

The Nicholson's blowflies diffusive equation together with the food-limited diffusive equations [22, 40, seem to be the first scalar models coming from applications where untypical behavior in the form of non-monotone non-oscillating wavefronts is established analytically and also observed in numerical experiments. Among previous studies, we would like to mention an illustrative example in [10] of the Mackey-Glass type diffusive equation with a single delay and with a piece-wise linear birth function.

Theorem 1 will be proved in the next section within the framework of the singular perturbation theory developed by Faria et al in 12, 13, 14. First, in Subsection 2.1. we analyze the unique heteroclinic solution $u_{*}(t)$ of equation (1). We show that the asymptotic Dirichlet series approximating $u_{*}(t)$ at $-\infty$ is uniformly convergent on a sufficiently long time interval. Then we use these approximations to detect parameters $(\tau, p)$ for which $u_{*}(t)$ is a non-monotone (but eventually monotone at $\pm \infty$ ) and non-oscillating solution of (1). Next, in Subsection 2.2, we extend the aforementioned properties of $u_{*}(t)$ on the wavefront profiles $\phi_{c}(t)$ for all sufficiently large speeds $c$. The key technical result of this subsection is Lemma 15 which excludes the existence of profiles $\phi_{c}(t)$ slowly oscillating around the steady state $u=\ln p$ and super-exponentially converging to $u=\ln p$ at $+\infty$. Lemma 15 shows that the Mallet-Paret-Cao-Arino theory of super-exponential solutions [2, 6, 30] for scalar equations can be extended for some classes of second order 


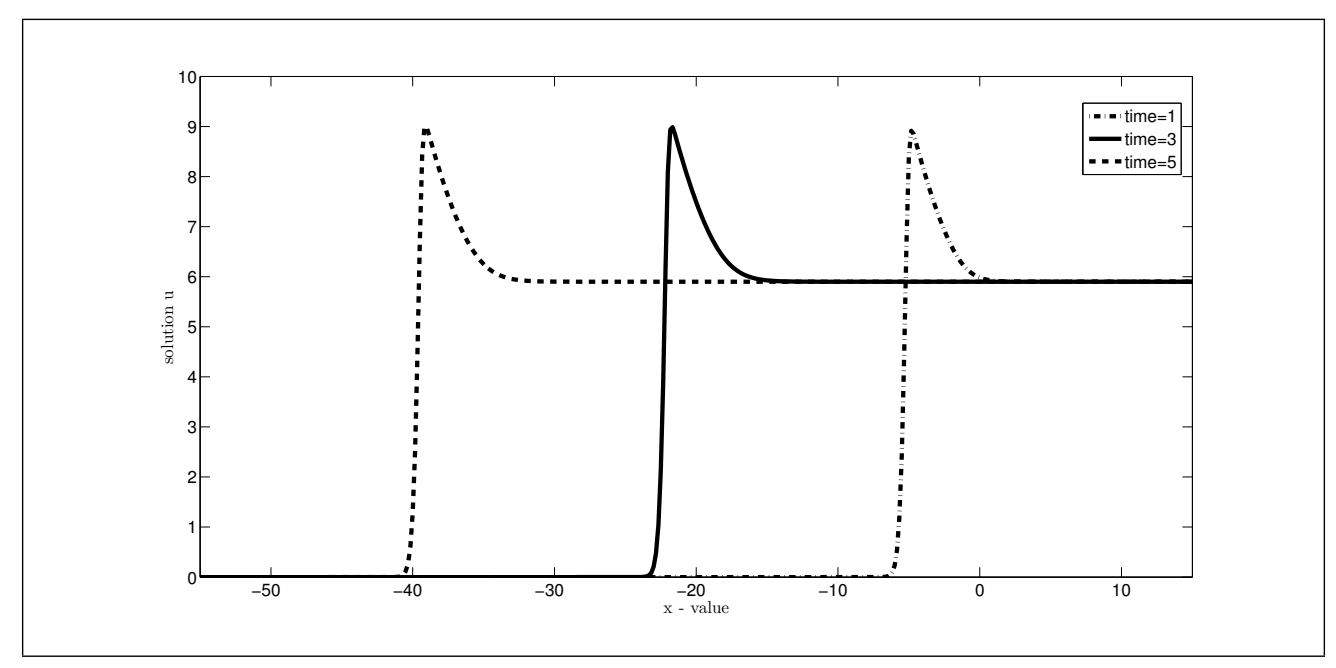

Figure 3: Numerical approximations of the minimal wavefront for equation 2 with $\tau=0.07, p=365$.

delay differential equations. Lemma 15 also helps to show that, in difference with the monotone wavefronts, eventually monotone wavefronts are linearly determined:

Theorem 3. Let $u=\phi(x+c t)$ be a wavefront for equation (2). Then the profile $\phi(t)$ is eventually monotone at $+\infty$ if and only if the characteristic function $\chi_{+}(z, c)=z^{2}-$ $c z-1-P e^{-z c \tau}, P:=\ln p-1$, at the equilibrium $u=\ln p$ has at least one negative zero.

Note that, by [42, Theorem 1], the leading edge of the profile $\phi(t)$ is strictly increasing till its first intersection with the equilibrium level $u=\ln p$. Eventual monotonicity criterion of Theorem 3 complements previous information concerning the shapes of waves for the Nicholson's diffusive equation, cf. [16, 27, 42. It is worth mentioning that our proofs use in an essential way several specific characteristics of the blowflies birth function (in particular, its unimodal form and the negativity of its Schwarz derivative). There are various other monostable population models with unimodal birth functions (e.g., see [3, Table 1], the cases with overcompensating density dependence). We believe that the problem of the nm-waves in these models can be approached by using techniques from the present work. For certain, this does not mean that each population model with overcompensating density dependence [3] necessarily possesses a nm-wave.

Eventual monotonicity criterion of Theorem 3 is proved in Section 2.3 as Corollary 18. We did not succeed to demonstrate this result by using well-known methods of upper and lower solutions or global continuation of waves (these methods were quite efficient in establishing criteria of wave's monotonicity on $\mathbb{R}$ [11, 16, 41]). Instead, we have combined the above mentioned Lemma 15 with wavefront's existence and oscillation results from [42, 43] (stated as Proposition 19 in Appendix). With such an approach, the main technical difficulty was establishing a connection between two different series of conditions (the first one given in Theorem 3 and the second one given in Proposition 19. The required relation (in the form of somewhat cumbersome inequality (22)) is proved in Appendix as Lemma 21. 


\section{Existence of non-monotone and non-oscillating wavefronts}

\subsection{On the approximation of the heteroclinic connections for the blowflies equation}

In this subsection, we establish several key properties of the positive heteroclinic connections to the Nicholson's blowflies delayed equation

$$
u^{\prime}(t)=-u(t)+f(u(t-\tau)), \quad f(u)=p u e^{-u}, \quad p>1 .
$$

The existence and uniqueness of these connections was previously demonstrated in 13 , [15, yet the mentioned works did not provide acceptable analytical tools to approximate the unique heteroclinic solution $u^{*}(t)$ (normalized at $-\infty$ ) on a given time interval. Sufficiently sharp approximations are however necessary to prove the existence of the nm-waves, cf. 22. To obtain such an approximation, we are using here the asymptotic Dirichlet series representing $u^{*}(t)$ at $-\infty$. It can be deduced from the Murovtsev theory 32 that this series is uniformly converging on some infinite interval $(-\infty, s)$. In the next theorem we are trying to find $s$ as large as possible by realizing a direct estimation of the Dirichlet series coefficients (in [32, the method of majorization of $u^{*}(t)$ by analytic functions was used).

Theorem 4. Suppose that $p>1, \tau>0$, and $\epsilon \in\left(0, e^{\mu \tau}-1\right)$. Then equation (4) has a unique (up to translation) positive solution $u^{*}(t)$ defined for all $t \in \mathbb{R}$ satisfying $u^{*}(-\infty)=0$. In addition, $u^{*}(t)$ is bounded, $\liminf _{t \rightarrow+\infty} u^{*}(t)>0$, and $u^{*}(t)$ is a real analytic function. Moreover, the solution $u^{*}(t)$ at $-\infty$ can be represented, modulo an appropriate shift in time, by the Dirichlet series

$$
u^{*}(t)=e^{\mu t}+\bar{q}_{2} e^{2 \mu t}+\bar{q}_{3} e^{3 \mu t}+\cdots+\bar{q}_{n} e^{n \mu t}+\ldots, \quad t \rightarrow-\infty,
$$

absolutely and uniformly converging on closed subsets of the interval

$$
(-\infty, T):=\left(-\infty, \tau+\mu^{-1} \ln \left[\frac{\epsilon}{1+\epsilon} \ln \left(1+\frac{1}{\left|\bar{q}_{2}\right|(1+\epsilon)}\right)\right]\right) .
$$

The coefficients $\bar{q}_{j}, j \geq 2$, alternate in sign and can be calculated recursively from equation (4). In particular, $\bar{q}_{2}=-p e^{-2 \mu \tau} / \chi(2 \mu)<0, \bar{q}_{3}=p\left(0.5-2 \bar{q}_{2}\right) e^{-3 \mu \tau} / \chi(3 \mu)>0$, with $\chi$ defined in (3).

Proof. We look for an analytic solution $u=u(t)$ of equation (4) in the form

$$
u(t)=q_{1} e^{\mu t}+q_{2} e^{2 \mu t}+q_{3} e^{3 \mu t}+\cdots+q_{n} e^{n \mu t}+\ldots,
$$

with some $q_{1}>0$. After comparing the coefficients of $e^{(n+1) \mu t}$ in both sides of the next equation (obtained from (4) by using a series representation for $f(u)$ )

$u^{\prime}(t)+u(t)-p u(t-\tau)=-p\left(u^{2}(t-\tau)-\frac{u^{3}(t-\tau)}{2 !}+\cdots+(-1)^{n+1} \frac{u^{n+1}(t-\tau)}{n !}+\ldots\right)$

we get the recurrent formula determining $q_{n+1}, n \geq 1$ :

$q_{n+1}=-\frac{p e^{-(n+1) \mu \tau}}{\chi((n+1) \mu)}\left[\sum_{i_{1}+i_{2}=n+1} q_{i_{1}} q_{i_{2}}-\frac{1}{2 !} \sum_{\substack{i_{1}+i_{2}+i_{3}=n+1 \\ 6}} q_{i_{1}} q_{i_{2}} q_{i_{3}}+\cdots+\frac{(-1)^{n+1}}{n !} q_{1}^{n+1}\right]$. 
The general term of the sum in brackets is

$$
A_{k}:=\frac{(-1)^{k}}{(k-1) !} \sum_{i_{1}+i_{2} \cdots+i_{k}=n+1} q_{i_{1}} q_{i_{2}} \ldots q_{i_{k}}, \quad k \geq 2 .
$$

Assuming that $(-1)^{j+1} q_{j}>0$ for all $j \leq n$, we find that $\operatorname{sign} A_{k}$ is

$$
\operatorname{sign} \frac{(-1)^{n+1+k+k}}{(k-1) !} \sum_{i_{1}+i_{2} \cdots+i_{k}=n+1}(-1)^{i_{1}+1} q_{i_{1}}(-1)^{i_{2}+1} q_{i_{2}} \ldots(-1)^{i_{k}+1} q_{i_{k}}=\operatorname{sign}(-1)^{n+1} .
$$

Therefore $\operatorname{sign} q_{n+1}=-\operatorname{sign} A_{k}=\operatorname{sign}(-1)^{n}$. Notice here that $\chi((n+1) \mu) \geq \chi(2 \mu)>0$ for all $n \in \mathbb{N}$.

Suppose now that $\left|q_{j}\right| \leq q_{1}:=\sigma$ for each $j=1, \ldots, n$. Then, invoking elementary combinatorics together with the Egorychev method [9], we find that, for each $\epsilon>0$,

$$
\begin{gathered}
\left|q_{n+1}\right| \leq \frac{p e^{-(n+1) \mu \tau}}{\chi((n+1) \mu)}\left[\sum_{i_{1}+i_{2}=n+1} \sigma^{2}+\frac{1}{2 !} \sum_{i_{1}+i_{2}+i_{3}=n+1} \sigma^{3}+\cdots+\frac{\sigma^{n+1}}{n !}\right]= \\
\frac{p e^{-(n+1) \mu \tau}}{\chi((n+1) \mu)}\left[\sigma^{2}\left(\begin{array}{c}
n \\
1
\end{array}\right)+\frac{\sigma^{3}}{2 !}\left(\begin{array}{l}
n \\
2
\end{array}\right)+\cdots+\frac{\sigma^{n+1}}{n !}\left(\begin{array}{l}
n \\
n
\end{array}\right)\right]= \\
\frac{p e^{-(n+1) \mu \tau}}{\chi((n+1) \mu)} \frac{1}{2 \pi i} \oint_{|z|=\epsilon}(1+z)^{n}\left[\frac{\sigma^{2}}{z^{2}}+\frac{\sigma^{3}}{z^{3} 2 !}+\cdots+\frac{\sigma^{n+1}}{z^{n+1} n !}\right] d z \leq \\
\frac{p e^{-(n+1) \mu \tau}}{\chi((n+1) \mu)} \frac{1}{2 \pi i} \oint_{|z|=\epsilon} \frac{\sigma(1+z)^{n}}{z}\left[\frac{\sigma}{z}+\frac{\sigma^{2}}{z^{2} 2 !}+\cdots+\frac{\sigma^{n}}{z^{n} n !}+\cdots\right] d z \leq \\
\frac{\sigma p e^{-(n+1) \mu \tau}}{\chi((n+1) \mu)}\left(e^{\sigma / \epsilon}-1\right)(1+\epsilon)^{n} \leq \sigma\left|\bar{q}_{2}\right|\left(e^{\sigma / \epsilon}-1\right) e^{\mu \tau}\left(e^{-\mu \tau}(1+\epsilon)\right)^{n} \leq \sigma
\end{gathered}
$$

whenever $\left|\bar{q}_{2}\right|\left(e^{\sigma / \epsilon}-1\right)(1+\epsilon) \leq 1$ and $e^{-\mu \tau}(1+\epsilon)<1$. Thus, for each $\epsilon \in\left(0, e^{\mu \tau}-1\right)$, the Dirichlet series (6) with $q_{1}=\epsilon \ln \left(1+\left[\left|\bar{q}_{2}\right|(1+\epsilon)\right]^{-1}\right)$ converges absolutely and uniformly on each closed subset of $\left(-\infty, \tau-\mu^{-1} \ln (1+\epsilon)\right)$. Equivalently, the shifted series

$$
u^{*}(t):=u\left(t-\mu^{-1} \ln q_{1}\right)=e^{\mu t}+\frac{q_{2}}{q_{1}^{2}} e^{2 \mu t}+\frac{q_{3}}{q_{1}^{3}} e^{3 \mu t}+\cdots+\frac{q_{n}}{q_{1}^{n}} e^{n \mu t}+\ldots
$$

converges for all $t<T=\tau-\mu^{-1} \ln (1+\epsilon)+\mu^{-1} \ln q_{1}$. Hence, equation (4) has an analytic solution $u^{*}(t)$ defined and positive on some interval $\left(-\infty, T_{1}\right] \subset(-\infty, T)$. Clearly, integrating (4) step by step, we can extend this solution for all $t \in \mathbb{R}$. Next, the set $X:=C([-\tau, 0],(0,+\infty))$ is invariant with respect to the semi-flow $\Phi^{t}$ generated by (4) so that $u^{*}(t)>0$ for all $t \in \mathbb{R}$. Furthermore, since all solutions to (4) are uniformly persistent when $p>1$ [4, Theorem 2.4], the trajectory $\Phi^{t} u_{0}^{*}$, where $u_{0}^{*}(s)=u^{*}(s), s \in[-\tau, 0]$, has a compact $\omega$-limit set in $X$. In particular, $u^{*}(t)$ is bounded and $\lim \inf _{t \rightarrow+\infty} u^{*}(t)>0$. Then by the classical Nussbaum theorem [34, $u^{*}$ is a real analytic function on $\mathbb{R}$. Finally, the uniqueness of $u^{*}$ was established in the proofs of [13, Lemmas 6 and 8]. Alternatively, it can be deduced from the general uniqueness Theorem 3 in [1]. 
Remark 5. Suppose that either $1<p \leq e^{2}$ or $p>e^{2}$ and

$$
e^{-\tau}>P \ln \frac{P^{2}+P}{P^{2}+1}, \quad \text { where } P=\ln p-1 .
$$

Then [29, Theorem 2.1] implies that the positive solution $u^{*}(t)$ given in Theorem 4 converges at $+\infty: u^{*}(+\infty)=\ln p$.

Example 6. As in Example 2, take $\tau=0.07$ and $p=365$ and choose $\epsilon=2.2<e^{\mu \tau}-1=$ $9.536 \ldots$ Then Theorem 4 provides the convergence interval $(-\infty, 0.079] \supset \mathbb{R}_{-}$for the series (5). In addition, in this particular case, $\left|\bar{q}_{j}\right|$ are decreasing which suggests the following estimates for $u^{*}(t)$ in the spirit of the alternating series test:

$$
u_{2}(t):=e^{\mu t}+\bar{q}_{2} e^{2 \mu t}<u^{*}(t)<e^{\mu t}=: u_{1}(t), \quad t \leq 0 .
$$

Our next result shows that this estimate is indeed true.

Theorem 7. Solution $u^{*}(t)$ described in Theorem 4 and normalized at $-\infty$ by (5) satisfies (8).

Proof. In view of Theorem 4, the inequalities (8) hold on some interval $(-\infty, L)$ with $L \leq 0$. It is easy to see that $u^{*}(t)<u_{1}(t)$ for all $t \in \mathbb{R}$. Indeed, suppose that $u_{1}(a)=$ $u^{*}(a)$ at some leftmost point $a$. Then $u_{1}^{\prime}(a) \leq\left(u^{*}\right)^{\prime}(a), u_{1}(a-\tau)>u^{*}(a-\tau)$, so that

$$
\begin{gathered}
0=\left(u^{*}\right)^{\prime}(a)+u^{*}(a)-f\left(u^{*}(a-\tau)\right)>\left(u^{*}\right)^{\prime}(a)+u^{*}(a)-p u^{*}(a-\tau)> \\
\left(u_{1}\right)^{\prime}(a)+u_{1}(a)-p u_{1}(a-\tau)=0,
\end{gathered}
$$

a contradiction. Hence $u^{*}(t)<e^{\mu t}$ for all $t \in \mathbb{R}$. In particular, $u^{*}(t)<1$ for all $t \leq 0$.

Next, we have that

$$
u_{2}^{\prime}(t)+u_{2}(t)-f\left(u_{2}(t-\tau)\right)<u_{2}^{\prime}(t)+u_{2}(t)-p\left(u_{2}(t-\tau)-u_{2}^{2}(t-\tau)\right)=p \bar{q}_{2} e^{3 \mu(t-\tau)}\left(2+\bar{q}_{2} e^{\mu(t-\tau)}\right) .
$$

Since $u_{2}(t)>0$ if and only if $1+\bar{q}_{2} e^{\mu t}>0$, we conclude that $u_{2}^{\prime}(t)+u_{2}(t)-f\left(u_{2}(t-\tau)\right)<0$ whenever $u_{2}(t)>0$.

We claim that actually the first inequality in $(8)$ also holds for all $t \leq 0$. Since $u^{*}(t)>0$ for all $t \in \mathbb{R}$, it suffices to prove it only for those $t<0$ for which $u_{2}(t)>0$. Arguing by contradiction again, suppose that $u_{2}(a)=u^{*}(a)>0$ at some leftmost point $a \leq 0$. Then $u_{2}^{\prime}(a) \geq\left(u^{*}\right)^{\prime}(a), u_{2}(a-\tau)<u^{*}(a-\tau)<1$ so that

$$
0=\left(u^{*}\right)^{\prime}(a)+u^{*}(a)-f\left(u^{*}(a-\tau)\right)<\left(u_{2}\right)^{\prime}(a)+u_{2}(a)-f\left(u_{2}(a-\tau)\right)<0,
$$

a contradiction. Observe here that the strict monotonicity of $f(x)=p x e^{-x}$ on the interval $[0,1]$ plays an essential role in the last argument.

We will also need the following property of $u^{*}(t)$ :

Lemma 8. $\left(u^{*}\right)^{\prime}(t)>0$ on some maximal interval $(-\infty, M) \subseteq \mathbb{R}$. Clearly, if $M=+\infty$, then $u^{*}(M)=\ln p$. If $M$ is finite, then $u^{*}(M)>\ln p$. 
Proof. By differentiating (5), we find that $\left(u^{*}\right)^{\prime}(t)=\mu e^{\mu t}(1+o(1))>0$ at $t=-\infty$, from which the existence of the above mentioned $M$ follows. Suppose that $M$ is finite, then $\left(u^{*}\right)^{\prime}(M)=0,\left(u^{*}\right)^{\prime \prime}(M) \leq 0,\left(u^{*}\right)^{\prime}(M-\tau)>0$. Since (4) implies the relation $\left(u^{*}\right)^{\prime \prime}(M)=f^{\prime}\left(u^{*}(M-\tau)\right)\left(u^{*}\right)^{\prime}(M-\tau)$, we obtain $f^{\prime}\left(u^{*}(M-\tau)\right) \leq 0$ so that $u^{*}(M-\tau) \geq$ 1. Clearly, if also $u^{*}(M-\tau) \geq \ln p$ then $u^{*}(M)>u^{*}(M-\tau) \geq \ln p$ and the lemma is proved in such a case. On the other hand, if $u^{*}(M-\tau) \in[1, \ln p)$ then the statement follows from the relation $u^{*}(M)=f\left(u^{*}(M-\tau)\right)>\ln p$.

Now, looking for wavefronts to 2 in the form

$$
u(t, x)=\phi(\sqrt{\epsilon} x+t), \quad \epsilon=c^{-2},
$$

we obtain the profile equation

$$
\epsilon \phi^{\prime \prime}(t)-\phi^{\prime}(t)-\phi(t)+f(\phi(t-\tau))=0 .
$$

Equation (9) was analyzed in 11, 13, 15, 16, 26, 27, 37, 42. Since the first derivative of $f(x)=p x e^{-x}$ is dominated by $f^{\prime}(0)=p,\left|f^{\prime}(x)\right| \leq p$ for all $x \geq 0$, the uniqueness (up to translation) of each semi-wavefront $\phi(t, \epsilon)$ to $(9)$ follows from [1, Theorem 7]. Next, by [37. or [42, Corollary 12] if $1<p \leq e$ (i.e. if $f(x)$ is monotone on $[0, \ln p]$ ) then $\phi^{\prime}(t, \epsilon)>0$ for all $t \in \mathbb{R}$. Now, when $p \in\left(e, e^{2}\right], f(x)$ is no longer monotone on $[0, \ln p]$. Nevertheless, in such a case $f^{\prime}(\ln p) \leq f^{\prime}(x) \leq f^{\prime}(0)$ for all $x \in[0, \ln p]$ and $f(x)$ satisfies the feedback condition on the interval $(f(p / e), p / e) \backslash \ln p$ (see condition (18) in Appendix). Therefore [16, 42. assure that if $e<p \leq e^{2}$ then $\phi(t, \epsilon)$ is either monotone or sine-like slowly oscillating around $\ln p$ at $+\infty$; moreover, the type of monotonicity/oscillation property is linearly determined. Similarly, [16. Theorem 2.5] implies that $\phi(t, \epsilon)$ is strictly increasing when $p>e^{2}$ and $p \tau e^{\tau-1} \leq 1$. Next, it is easy to see that the characteristic equation $\chi_{+}(z, \epsilon):=\epsilon z^{2}-z-1-P e^{-z \tau}$ does not have real negative roots when $P \tau e^{1+\tau} \geq 1$. Then [42, Lemma 25] implies that $\phi(t, \epsilon)$ cannot be eventually monotone if $P \tau e^{1+\tau} \geq 1$. Hence, we have established the following result:

Lemma 9. Suppose that equation (2) has a nm-wave. Then necessarily $p>e^{2}, P \tau e^{1+\tau}<$ 1 and $p \tau e^{\tau-1}>1$. In particular, $\tau \in\left(0, \tau_{*}\right)$ where $\tau_{*}=0.278 \ldots$ solves equation $\tau e^{1+\tau}=1$.

Corollary 10. If equation (2) has a nm-wave then $e^{-\mu \tau}<0.5$ and $\bar{q}_{2} \in(-1,0)$.

Proof. Indeed, $\mu+1=p e^{-\mu \tau}>e^{1-(\mu+1) \tau} / \tau$. Set $\rho=\tau(\mu+1)$, then $\rho>e^{-\rho+1}$ implying that $\rho>1$. Consequently, $-\tau \mu<\tau-1$ and $e^{-\tau \mu}<e^{-1+\tau}<e^{-1+\tau_{*}}=0.48 \ldots$, $-\bar{q}_{2}=p e^{-2 \mu \tau} / \chi(2 \mu)=p e^{-2 \mu \tau} /\left(\mu+p\left(e^{-\mu \tau}-e^{-2 \mu \tau}\right)\right)<e^{-\mu \tau} /\left(1-e^{-\mu \tau}\right)=0.94 \ldots$

In the next stage of our proof, we continue to analyze the heteroclinic solution $u^{*}(t)$ given in Theorem 4. This time, we will take parameters $(\tau, p)$ as in Lemma 9 which will have important consequences for the form and estimates of $u^{*}(t)$. For example, in such a case, due to Corollary 10 the lower bound $u_{2}(t)$ in $\sqrt{8}$ is positive for all $t \leq 0$. Especially we will be interested in the oscillation properties (around the equilibrium $\ln p$ ) of $u^{*}(t)$. Since $u^{*}(t)$ is a real analytic function on $\mathbb{R}$, the set of all solutions of equation $u^{*}(t)=\ln p$ is either an empty set or can be represented as a strictly increasing sequence $\mathcal{S}=\left\{t_{j}\right\}$ of positive numbers. By the same reason, if the sequence $\mathcal{S}$ is infinite, it should 
converge to $+\infty$. Our next goal is to prove that $t_{j}$ are simple zeros of $u^{*}(t)-\ln p$ and that $t_{j+1}-t_{j}>\tau$. The main complication here consists in the fact that the birth function $f(x)=p x e^{-x}$ for $p>16.99 \ldots$ does not satisfy the feedback condition [18), cf. [16, 42]. Our arguments below are inspired by the classical theory of J. Mallet-Paret [30].

Lemma 11. Suppose that $p>e^{2}$ and $P \tau e^{1+\tau}<1$. Then inequality (7) is satisfied so that $u^{*}(+\infty)=\ln p$ and, in fact, $u^{*}(t)$ is eventually monotone solution. Furthermore, the above defined sequence $\left\{t_{j}\right\}$ has at most a finite number of elements, $t_{1}<t_{2}<\cdots<t_{m}$, $m \in \mathbb{N}$ and $t_{j+1}-t_{j}>\tau,\left(u^{*}\right)^{\prime}\left(t_{j}\right) \neq 0$ for each such $t_{j}$.

PROOF. It is easy to check that the smooth curves $\gamma_{1}, \gamma_{2} \subset \mathbb{R}_{+}$defined by equations $P \tau e^{1+\tau}=1$ and $e^{-\tau}=P \ln \frac{P^{2}+P}{P^{2}+1}$, respectively, intersect at some point $\left(\tau_{0}, P_{0}\right)$ if and only if $\tau_{0}$ satisfies equation $e^{\tau e}\left(1+\tau^{2} e^{2(1+\tau)}\right)=\tau e^{1+\tau}+1$. However, a straightforward comparison of the Taylor coefficients in this equation shows that $e^{\tau e}>\tau e^{1+\tau}+1$ for all positive $\tau$. Hence, $\gamma_{1} \cap \gamma_{2}=\emptyset$. Actually, an easy inspection shows that the domain bounded by $\gamma_{1}$ and the coordinate axes lies inside the domain bounded by $\gamma_{2}$ and the coordinate axes.

Now, suppose that $t_{1}$ is the moment of the first intersection of the graph of $u^{*}(t)$ with the line $u=\ln p$. Then $\left(u^{*}\right)^{\prime}\left(t_{1}\right)>0$ in view of Lemma 8. Suppose next that $t_{2}$ is the moment of the second intersection of $u^{*}(t)$ with the line $u=\ln p$ and $u^{*}\left(s_{1}\right):=$ $\max \left\{u^{*}(t), t \in\left[t_{1}, t_{2}\right]\right\}$. Then $\left(u^{*}\right)^{\prime}\left(s_{1}\right)=0,\left(u^{*}\right)^{\prime}\left(t_{2}\right) \leq 0$, from which we obtain $\ln p<u^{*}\left(s_{1}\right)=f\left(u^{*}\left(s_{1}-\tau\right)\right), \ln p=u^{*}\left(t_{2}\right) \geq f\left(u^{*}\left(t_{2}-\tau\right)\right)$. In the case when $t_{2}-t_{1} \leq \tau$ we have, in addition, that $\ln p \geq u^{*}\left(t_{2}-\tau\right)>u^{*}\left(s_{1}-\tau\right)$. Consider first the situation when $\ln p>u^{*}\left(t_{2}-\tau\right)$. Then $\ln p \geq f\left(u^{*}\left(t_{2}-\tau\right)\right)$ implies that $u^{*}\left(t_{2}-\tau\right) \leq \min f^{-1}(\ln p)$. On the other hand $\ln p<u^{*}\left(s_{1}\right)=f\left(u^{*}\left(s_{1}-\tau\right)\right)$ implies that $u^{*}\left(s_{1}-\tau\right)>\min f^{-1}(\ln p)$. Thus $u^{*}\left(t_{2}-\tau\right)<u^{*}\left(s_{1}-\tau\right)$, a contradiction. Finally, suppose that $\ln p=u^{*}\left(t_{2}-\tau\right)=$ $u^{*}\left(t_{2}\right)$. Then $\left(u^{*}\right)^{\prime}\left(t_{2}\right)=0$ implying the following contradictory relation $\left(u^{*}\right)^{\prime \prime}\left(t_{2}\right)=$ $f^{\prime}\left(u^{*}\left(t_{2}-\tau\right)\right)\left(u^{*}\right)^{\prime}\left(t_{2}-\tau\right)<0$. The above discussion shows that $t_{2}-t_{1}>\tau$ and that $\left(u^{*}\right)^{\prime}\left(t_{2}\right)<0$.

By our convention, $u^{*}(t)<\ln p$ for $t \in\left(t_{2}, t_{3}\right)$ and $u^{*}\left(s_{2}\right)=\min \left\{u^{*}(t), t \in\left[t_{2}, t_{3}\right]\right\}$ for some leftmost $s_{2} \in\left(t_{2}, t_{3}\right)$. Again, $\ln p>u^{*}\left(s_{2}\right)=f\left(u^{*}\left(s_{2}-\tau\right)\right)$. Since $u^{*}\left(s_{2}\right)<$ $u^{*}\left(s_{2}-\tau\right)$, the latter implies that $u^{*}\left(s_{2}-\tau\right)>\ln p$ and, consequently, that $s_{2}-t_{2} \leq \tau$. Therefore, using the variation of constant formula, we get

$u^{*}\left(s_{2}\right)=e^{-\left(s_{2}-t_{2}\right)} \ln p+\int_{t_{2}}^{s_{2}} e^{-\left(s_{2}-s\right)} f\left(u^{*}(s-\tau)\right) d s>e^{-\tau} \ln p \geq e^{-\tau_{*}} \ln e^{2}=1.51 \ldots>1$.

In particular, $u^{*}(t)>1$ for all $t \in\left(t_{2}, t_{3}\right)$. Now, since $\left(u^{*}\right)^{\prime}\left(t_{3}\right) \geq 0$, we find that $\ln p=u^{*}\left(t_{3}\right) \leq f\left(u^{*}\left(t_{3}-\tau\right)\right)$. This implies that $u^{*}\left(t_{3}-\tau\right) \leq \ln p$. Suppose that $u^{*}\left(t_{3}-\tau\right)=\ln p$, then obviously $t_{3}-\tau=t_{2}$ (since $t_{1}<t_{1}+\tau<t_{2}<t_{3}$ are the consecutive zeros of $\left.u^{*}(t)-\ln p\right)$ and $\left(u^{*}\right)^{\prime \prime}\left(t_{3}\right)=f^{\prime}(\ln p)\left(u^{*}\right)^{\prime}\left(t_{2}\right)>0$, a contradiction. Consequently, $u^{*}\left(t_{3}-\tau\right)<\ln p,\left(u^{*}\right)^{\prime}\left(t_{3}\right)>0$ and $t_{3}-t_{2}>\tau$.

Next, $u^{*}(t)>\ln p$ for $t \in\left(t_{3}, t_{4}\right)$ and $\left(u^{*}\right)^{\prime}\left(t_{4}\right) \leq 0$. Thus $\ln p=u^{*}\left(t_{4}\right) \geq f\left(u^{*}\left(t_{4}-\right.\right.$ $\tau)$ ) from where $u^{*}\left(t_{4}-\tau\right) \geq \ln p$ (here we are using the inequality $u^{*}(t)>1$ for $t \in$ $\left.\left[t_{1}, t_{4}\right]\right)$. Suppose that $u^{*}\left(t_{4}-\tau\right)=\ln p$, then again we get $t_{4}-\tau=t_{3}$ and $\left(u^{*}\right)^{\prime \prime}\left(t_{4}\right)=$ $f^{\prime}(\ln p)\left(u^{*}\right)^{\prime}\left(t_{3}\right)<0$, a contradiction. Consequently, $u^{*}\left(t_{4}-\tau\right)>\ln p,\left(u^{*}\right)^{\prime}\left(t_{4}\right)<0$ and $t_{4}-t_{3}>\tau$. Clearly, the above described inductive steps can be continued to include all points $t_{j}$. 
Finally, we will prove that the sequence $\left\{t_{j}\right\}$ is finite. Linearizing (1) at the equilibrium $\ln p$, we find the associated characteristic function $\chi_{+}(z):=z+1+P e^{-z \tau}$. After a straightforward computation, we find that $\chi_{+}(z)$ has exactly two different real roots $z_{2}<z_{1}<0$ if and only if $P \tau e^{1+\tau}<1$. Moreover, in such a case, each other root $z_{j}=\alpha_{j}+i \beta_{j}, \beta_{j} \geq 0, j>2$, satisfies the inequalities $\alpha_{j}<z_{2}$ and $\beta_{j}>2 \pi / \tau$, see [30, Theorem 6.1]. In particular, the steady state $u=\ln p$ is exponentially stable. We claim that $u^{*}(t)$ is eventually monotone at $+\infty$ if $P \tau e^{1+\tau}<1$. Indeed, otherwise, it is easy to see that the sequence $\left\{t_{j}\right\}$ is infinite so that $u^{*}(t)$ exponentially converges to the equilibrium $\ln p$ and slowly oscillates around it. Then in view of Yulin Cao results [6. Theorem 3.4] on super-exponential solutions, there is a zero $z_{j}=\alpha_{j}+i \beta_{j}, j \in \mathbb{N}$, of $\chi_{+}(z)$ and $C \neq 0, \delta>0, \theta \in \mathbb{R}$, such that

$$
u^{*}(t)-\ln p=C e^{\alpha_{j} t} \cos \left(\beta_{j} t+\theta\right)+O\left(e^{\left(\alpha_{j}-\delta\right) t}\right), \quad t \rightarrow+\infty .
$$

Now, if $z_{j}$ is not a real root, the above representation implies that the distance between large adjacent zeros of $u^{*}(t)-\ln p$ is less than $\tau / 2$, a contradiction. Hence

$$
u^{*}(t)=\ln p+C_{*} e^{z_{j} t}+O\left(e^{\left(z_{j}-\delta\right) t}\right), \quad t \rightarrow+\infty,
$$

where $j \in\{1,2\}, z_{j}<0, C_{*} \neq 0$. This completes the proof of Lemma 11

Importantly, the sequence $\mathcal{S}$ can be non-empty for certain parameters $p, \tau$. The next result shows that generally $m \geq 1$ in Lemma 11. It is an open question, however, whether there exist $p, \tau$ for which $m \geq 2$.

Corollary 12. In addition to the assumptions of Lemma 11, suppose that $\zeta>\ln p$ where $\zeta$ was defined in Theorem 1. Then the solution $u^{*}(t)$ is eventually monotone at $+\infty$ and is non-monotone on $\mathbb{R}$. Moreover, $\max _{\mathbb{R}} u^{*}(t)>\zeta$.

Proof. Indeed, after using the variation of constant formula on $[0, \tau]$ and monotonicity of $f(u)$ on the interval $[0,1]$, we find that

$$
\begin{gathered}
u^{*}(\tau)=u^{*}(0) e^{-\tau}+\int_{0}^{\tau} e^{s-\tau} f\left(u^{*}(s-\tau)\right) d s>u_{2}(0) e^{-\tau}+\int_{0}^{\tau} e^{s-\tau} f\left(u_{2}(s-\tau)\right) d s> \\
u_{2}(0) e^{-\tau}+p \int_{0}^{\tau} e^{s-\tau} u_{2}(s-\tau) e^{-u_{1}(s-\tau)} d s= \\
\left(1+\bar{q}_{2}\right) e^{-\tau}+p \int_{-\tau}^{0} e^{\mu s} e^{s}\left(1+\bar{q}_{2} e^{\mu s}\right) \exp \left(-e^{\mu s}\right) d s=\zeta .
\end{gathered}
$$

Finally, recall that $\zeta>\ln p$ for the parameters $p=365, \tau=0.07$, see Example 2 .

\subsection{Proof of Theorem 1}

The key relation between $u^{*}(t)$ and $\phi(t, \epsilon)$ is given in the next assertion.

Proposition 13. Let all the conditions of Corollary 12 be satisfied. Then $\phi(+\infty, \epsilon)=$ $\ln p$ for all sufficiently small $\epsilon>0$. Moreover, $\lim _{\epsilon \rightarrow 0^{+}} \phi(t, \epsilon)=u^{*}(t)$ uniformly on $\mathbb{R}$ (possibly, after an appropriate translation of the wavefronts). In particular, the wave profiles $u=\phi(t, \epsilon)$ are not monotone for all small $\epsilon>0$. 
Proof. First, suppose that the characteristic equation $\chi(z)=0$ does not have roots on the imaginary axis. Then, in view of the uniqueness of semi-wavefronts in the Nicholson's blowflies diffusive equation, the statement concerning the uniform convergence is a direct consequence of [13, Theorem 1]. Now, if the zero equilibrium of equation (1) is not hyperbolic, the same conclusion follows from Theorem 3.8 in [14].

To complete the proof of Theorem 1 we have to extend the eventual monotonicity property of $\phi(t, 0)=u^{*}(t)$ established in Lemma 11 on the profiles $\phi(t, \epsilon)$ for all sufficiently small $\epsilon>0$. Observe that, taking into account Proposition 13 and using 31 , Theorem 2.1] we obtain (see [42, pp. 2321-2322] for more detail) that $\phi(t, \epsilon)$ is either eventually monotone or slowly oscillating around $\ln p$ at $+\infty$ in the following sense:

Definition 14. Set $\mathbb{K}=[-\tau, 0] \cup\{1\}$. For any $v \in C(\mathbb{K}) \backslash\{0\}$ we define the number of sign changes by

$\operatorname{sc}(v)=\sup \left\{k \geq 1\right.$ : there are $t_{0}<\cdots<t_{k}, t_{j} \in \mathbb{K}$, such that $v\left(t_{i-1}\right) v\left(t_{i}\right)<0$ for $\left.i \geq 1\right\}$.

We set $\operatorname{sc}(v)=0$ if $v(s) \geq 0$ or $v(s) \leq 0$ for $s \in \mathbb{K}$. Next, for a smooth function $\psi:\left[T_{0},+\infty\right) \rightarrow \mathbb{R}$ and a real number $\kappa$, we will write $\left(\bar{\psi}_{t}\right)(s)=\psi(t+s)-\kappa$ if $s \in[-\tau, 0]$, and $\left(\bar{\psi}_{t}\right)(1)=\psi^{\prime}(t)$. We will say that $\psi(t)$ is slowly oscillating around $\kappa$ on a connected interval $\mathfrak{J} \subset\left[T_{0}+\tau,+\infty\right)$ if the following conditions are satisfied:

(d1) $\psi(t)$ oscillates around $\kappa$ and

(d2) for each $t \in \mathfrak{J}$, it holds that either $s c\left(\bar{\psi}_{t}\right)=1$ or $\operatorname{sc}\left(\bar{\psi}_{t}\right)=2$.

Therefore our immediate goal is to demonstrate that $\phi(t, \epsilon)$ is not a slowly oscillating solution of equation (9) whenever all the conditions of Corollary 12 are satisfied. We have already solved a similar problem, proving eventual monotonicity of $u^{*}(t)$ in Lemma 11 . However, trying to argue as at the end of the proof of Lemma 11, we will regret the lack of an analog of the Mallet-Paret-Cao-Arino theory 2, 6, 30, of super-exponential solutions (i.e. solutions converging to their finite limits at $\infty$ faster than any exponential) for the second order delay differential equations. The key idea of this theory was concisely described by Ovide Arino: "Surprisingly, the proof [...] is based on quite an elementary although very neat observation, which is, essentially, if you assume a solution has a rapid decay from $t$ to $t+\tau$, it indicates rapid oscillations before $t$ ", see [2, p. 178]. In our next lemma, we contribute to the analysis of super-exponential solutions by developing this idea for second order delay differential equations (at a level of generality sufficient for our purposes).

Lemma 15. Let $y(t), y(+\infty)=0$, slowly oscillate around 0 and satisfy equation

$$
y^{\prime \prime}(t)-A y^{\prime}(t)-B y(t)-C(t) y(t-\tau)=0, \quad t \in \mathbb{R},
$$

where $A, B>0$ and continuous function $C(t)$ converges to a positive number $C$ at $+\infty$. Suppose also that the set of zeros of $y^{\prime}(t)$ does not contain a non-degenerate interval. Then $y(t)$ is not a super-exponentially decaying (i.e. small) solution.

Proof. Arguing by contradiction, suppose that equation (11) has a slowly oscillating small solution $y(t)$. After realizing the change of variables $y(t)=e^{r t} z(t)$, where $r$ is a 
negative root of equation $r^{2}-A r-B=0$, we obtain the delay differential equation for $z$ of the same type as (11) (i.e. $a>0, c(+\infty)>0$ ) except that now $B=0$ :

$$
y^{\prime \prime}(t)-a y^{\prime}(t)-c(t) y(t-\tau)=0, \quad t \in \mathbb{R} .
$$

Obviously, $z(t)=e^{-r t} y(t)$ is super-exponentially decaying at $+\infty$. It is easy to check that, being a small solution, $z(t)=e^{-r t} y(t)$ with $r<0$ shares the slow oscillation property of $y(t)$. We will arrive to a contradiction by supposing the existence of a slowly oscillating small solution $y(t)$ of equation (12).

Hence, arguing by contradiction, suppose that equation $(12)$ has a slowly oscillating small solution $y(t)$. In our subsequent argumentation, we will use the notation $y_{t}(s)=$ $y(t+s), s \in[-\tau, 0]$ so that $y_{t} \in C[-\tau, 0]$ and $\left\|y_{t}\right\|=\max \{|y(t+s)|, s \in[-\tau, 0]\}$. It is easy to see that the 'smallness' of $y(t)$ implies the existence of an increasing sequence $\left\{t_{j}\right\}, t_{j} \rightarrow+\infty$, such that $\left\|y_{t_{j}+2 \tau}\right\| /\left\|y_{t_{j}+\tau}\right\| \rightarrow 0$ as $j \rightarrow+\infty$ (since $y(t)$ oscillates, we have that $y_{t} \neq 0$ for each $t$ ).

Set $z_{j}(t)=y\left(t_{j}+t\right) /\left\|y_{t_{j}+\tau}\right\|$, clearly $\left|z_{j}(t)\right| \leq 1$ for $t \in[0, \tau]$ and $\left|z_{j}\left(\xi_{j}\right)\right|=1$ for some $\xi_{j} \in[0, \tau)$. Without loss of generality, we can further assume that $z_{j}\left(\xi_{j}\right)=1$ and that $\xi_{j} \rightarrow \xi_{*}$ for some $\xi_{*} \in[0, \tau]$. Due to our choice of $\left\{t_{j}\right\}$, we also find that $z_{j}(t) \rightarrow 0$ uniformly on the interval $[\tau, 2 \tau]$. In addition, $z_{j}(t)$ is a slowly oscillating small solution of the differential equation

$$
z^{\prime \prime}(t)-a z^{\prime}(t)-c_{j}(t) z(t-\tau)=0, \quad t \in \mathbb{R}, \text { where } c_{j}(t):=c\left(t+t_{j}\right) \rightarrow c_{*}:=C e^{-r \tau}>0 .
$$

We will say that an interval $(p, q)$ is a maximal complete interval of monotonicity for some $z_{j}$ if $z_{j}(t)$ is monotone on $(p, q), z_{j}^{\prime}(p)=z_{j}^{\prime}(q)=0$ and $(p, q)$ is not properly contained in a larger interval with the same properties. Then there exists some $r_{0} \in(p, q)$ such that $\left.0 \neq \mid z_{j}^{\prime}\left(r_{0}\right)\right) \mid=\max \left\{\left|z_{j}^{\prime}(s)\right|, s \in(p, q)\right\}$ and $z_{j}^{\prime \prime}\left(r_{0}\right)=0$. Thus

$$
\operatorname{sign} z_{j}\left(r_{0}-\tau\right)=\operatorname{sign}\left(-a z_{j}^{\prime}\left(r_{0}\right) / c_{j}\left(r_{0}\right)\right)=-\operatorname{sign} z_{j}^{\prime}\left(r_{0}\right)
$$

so that $z_{j}(t)$ can have at most 3 maximal complete intervals of monotonicity on each interval of length $\tau$ (consequently, at most 5 intervals of monotonicity). As a consequence, we can find a subsequence $\left\{z_{j_{i}}(t)\right\}$ of $\left\{z_{j}(t)\right\}$ such that that all $z_{j_{i}}(t)$ have exactly the same number of intervals of monotonicity $\left(p_{k}^{\left(j_{i}\right)}, q_{k}^{\left(j_{i}\right)}\right)$ inside of each of the segments $[-\tau, 0],[0, \tau],[\tau, 2 \tau]$, while the sequences $p_{k}^{\left(j_{i}\right)}, q_{k}^{\left(j_{i}\right)}$ are converging to their respective limits $p_{k}, q_{k}$. Without loss of generality, we can also assume that every $z_{j_{i}}(t)$ does not change its sign on each of the intervals $\left(p_{k}^{\left(j_{i}\right)}, q_{k}^{\left(j_{i}\right)}\right)$. Furthermore, due tho the Helly selection theorem [5. p. 250], we can assume that there exists a piece-wise monotone function $z_{*}(t), t \in[0,2 \tau]$, such that $z_{*}(t)=0, t \in[\tau, 2 \tau]$ and $z_{j_{i}}(t) \rightarrow z_{*}(t)$ pointwise on $[0,2 \tau]$. In the sequel, to simplify the notation, we will denote by $z_{j}(t)$ also any subsequence of $\left\{z_{j}(t)\right\}$.

We claim that $z_{*}(t)=0$ almost everywhere on $[0, \tau]$. First, observe that the sequence of the total variations $\int_{\tau}^{2 \tau}\left|z_{j}^{\prime}(s)\right| d s$ of the smooth functions $z_{j}(t)$ on $[\tau, 2 \tau]$ converges to 0 . Then, by the Riesz theorem [5, p. 79], we can extract a subsequence $z_{j}^{\prime}(t)$ such that $z_{j}^{\prime}(t) \rightarrow 0$ almost everywhere on $[\tau, 2 \tau]$. Take some $s_{1}<s_{2}, s_{k} \in[\tau, 2 \tau], k=1,2$, such that $\lim _{j \rightarrow \infty} z_{j}^{\prime}\left(s_{k}\right)=0$. By integrating $(13)$, we find that

$$
z_{j}^{\prime}\left(s_{2}\right)=e^{a\left(s_{2}-s_{1}\right)} z_{j}^{\prime}\left(s_{1}\right)+\int_{13}^{s_{2}} e^{a\left(s_{2}-s\right)} c_{j}(s) z_{j}(s-\tau) d s .
$$


Passing to the limit $j \rightarrow+\infty$ in [14, we find that, for almost all $s_{1}<s_{2}, s_{k} \in[\tau, 2 \tau]$, $k=1,2$,

$$
\int_{s_{1}}^{s_{2}} e^{a\left(s_{2}-s\right)} z_{*}(s-\tau) d s=0,
$$

which proves our claim.

Now, fix some non-empty monotonicity interval $\left(p_{k}, q_{k}\right) \subset[0, \tau]$ mentioned before. Due to the claim of the previous paragraph, we know that there are $r_{1}, r_{2} \in\left(p_{k}, q_{k}\right)$ such that $\lim _{j \rightarrow \infty} z_{j}\left(r_{k}\right)=0$. Then we find immediately that $\int_{r_{1}}^{r_{2}}\left|z_{j}^{\prime}(t)\right| d t \rightarrow 0$ and $z_{j}(t) \rightarrow 0$ uniformly on $\left[r_{1}, r_{2}\right]$. Arguing now as in the previous paragraph and passing to subsequences if necessary, we can conclude that $z_{j}^{\prime}(t) \rightarrow 0$ almost everywhere on $\left(p_{k}, q_{k}\right)$. Hence, we have proved that there exists a finite set of points $\mathfrak{R}=\left\{p_{k}, q_{j}: p_{k}, q_{j} \in[0, \tau]\right\}$ such that $z_{j}(t) \rightarrow 0$ uniformly on each closed interval $\mathcal{J} \subset[0, \tau] \backslash \mathfrak{R}$. Consequently, $z_{j}^{\prime}(t) \rightarrow 0$ almost everywhere on $[0, \tau]$ and $\xi_{*} \in \mathfrak{R}$.

In the next stage, we will analyze the sequence $\left\{z_{j}(t)\right\}$ on the interval $[-\tau, 0]$. Set $\mathfrak{R}_{1}=\left\{p_{k}, q_{j}: p_{k}, q_{j} \in[-\tau, 0]\right\}$ and consider some closed interval $[\alpha, \beta] \subset[-\tau, 0] \backslash \mathfrak{R}_{1}$. For all sufficiently large $j$, the functions $z_{j}(t)$ have the same type of positivity and monotonicity on $[\alpha, \beta]$. We claim that we can choose a subsequence in such a way that $z_{j}(t) \rightarrow 0$ uniformly on $[\alpha, \beta]$. To be more specific, suppose, for example, that $z_{j}(t)$ are non-negative and decreasing on $[\alpha, \beta]$. If some subsequence of $\left\{z_{j}(s)\right\}$ converges to $+\infty$ for $s \in(\alpha, \beta)$, then clearly $z_{j}(t) \rightarrow+\infty$ uniformly on $[\alpha, s]$ while $z_{j}^{\prime}(t) \rightarrow 0$ almost everywhere on $[\alpha+\tau, s+\tau]$. But then, after taking some $s_{1}<s_{2}, s_{k} \in[\alpha+\tau, s+\tau]$, $k=1,2$, such that $\lim _{j \rightarrow \infty} z_{j}^{\prime}\left(s_{k}\right)=0$ and using (14), we immediately get a contradiction. This implies that the sequence $z_{j}(t)$ is bounded for each $t \in(\alpha, \beta)$. In fact, since we can slightly move the endpoints $\alpha, \beta$ of this interval, the sequences $z_{j}(\alpha), z_{j}(\beta)$ are also bounded. This allows us to conclude that $\left\{z_{j}(t)\right\}$ is uniformly bounded on $[\alpha, \beta]$. Consequently, by arguing as above and passing to subsequences if necessary, we find that $z_{j}(t) \rightarrow 0$ uniformly on $[\alpha, \beta]$ and $z_{j}^{\prime}(t) \rightarrow 0$ almost everywhere on $[\alpha, \beta]$. In other words, $z_{j}(t), z_{j}^{\prime}(t)$ have similar convergence properties on the intervals $[0, \tau]$ and $[-\tau, 0]$.

Therefore, in each $\delta$-neighborhood of $\xi_{*}$, we can find points $t_{1}<\xi_{*}<t_{2}, \theta_{j}$ such that $\lim z_{j}\left(t_{k}\right)=\lim z_{j}^{\prime}\left(t_{k}\right)=0, k=1,2, t_{1}<\theta_{j} \leq \xi_{j}<t_{2}$ (whenever $j$ is sufficiently large) and $z_{j}^{\prime}\left(\theta_{j}\right)=0, z_{j}^{\prime \prime}\left(\theta_{j}\right) \leq 0, z_{j}\left(\theta_{j}\right) \geq 1$. Similarly, the interval $\left[\xi_{*}-\tau+\delta, \xi_{*}-\tau+2 \delta\right]$ contains a point $t_{3}>t_{2}-\tau$ such that $\lim z_{j}\left(t_{3}\right)=\lim z_{j}^{\prime}\left(t_{3}\right)=0$.

But then, if $\delta \in(0,0.5)$, there are some points $s_{j}, S_{j}$ satisfying the inequalities $t_{1}<$ $s_{j}<\theta_{j}<S_{j}<t_{2}$ and the relations $z_{j}^{\prime}\left(s_{j}\right)>2, z_{j}^{\prime \prime}\left(s_{j}\right)=0, z_{j}^{\prime}\left(S_{j}\right)<-2, z_{j}^{\prime \prime}\left(S_{j}\right)=0$. Since $c_{j}\left(t_{j}\right) \rightarrow c_{*}>0$, we can also assume that $0<c_{j}(t)<2 c_{*}$. Consequently, in view of equation $(13)$,

$$
\begin{gathered}
z_{j}\left(\theta_{j}-\tau\right) \leq 0, \quad z_{j}\left(s_{j}-\tau\right)=-a z^{\prime}\left(s_{j}\right) / c_{j}\left(s_{j}\right)<-2 a / c_{j}\left(s_{j}\right)<-a / c_{*}<0, \\
z_{j}\left(S_{j}-\tau\right)=-a z^{\prime}\left(S_{j}\right) / c_{j}\left(S_{j}\right)>2 a / c_{j}\left(S_{j}\right)>a / c_{*}>0 .
\end{gathered}
$$

Now, since $\xi_{*}-\delta-\tau<\theta_{j}-\tau<S_{j}-\tau<\xi_{*}+\delta-\tau<t_{3}<\xi_{*}+2 \delta-\tau$, for each $j$ big enough we can find points $M_{j}, T_{j} \in\left[\theta_{j}-\tau, t_{3}\right]$ such that $M_{j}<T_{j}, z_{j}\left(M_{j}\right)=\max \left\{z_{j}(s), s \in\right.$ $\left.\left[\theta_{j}-\tau, t_{3}\right]\right\}, z_{j}^{\prime}\left(T_{j}\right)<-2 a /\left(3 c_{*}\right), z_{j}^{\prime \prime}\left(T_{j}\right)=0$. Thus $z_{j}\left(T_{j}-\tau\right)=-a z^{\prime}\left(T_{3}\right) / c_{j}\left(T_{3}\right)>0$. Finally, consider four points $T_{j}-\tau<s_{j}-\tau<M_{j}<T_{j}$. Since $z_{j}\left(T_{j}-\tau\right)>0$, $z_{j}\left(s_{j}-\tau\right)<0, z_{j}\left(M_{j}\right)>0, z_{j}^{\prime}\left(T_{j}\right)<0$, we conclude that $\operatorname{sc}\left(\left(\bar{z}_{j}\right)_{T}\right)>2$ so that $z_{j}(t)$ does not slowly oscillate around 0 . The obtained contradiction completes the proof of Lemma 15. 
Corollary 16. Assume all the conditions of Corollary 12, Then there exists $\epsilon_{0}>0$ such that the solution $\phi(t, \epsilon)$ is eventually monotone at $+\infty$ for each $\epsilon \in\left[0, \epsilon_{0}\right]$.

Proof. After linearizing (9) at the equilibrium $\phi(t)=\ln p$, we find the related characteristic equation

$$
\chi_{+}(z, \epsilon)=\epsilon z^{2}-z-1-P e^{-z \tau}=0 .
$$

If $P \tau e^{1+\tau}<1$, it follows from [16, Lemma 1.1] and [43, Lemma 2.1] that there are $\delta>0$ and $\epsilon_{1}>0$ such that 150 has in the half-plane $\Re z>z_{2}-2 \delta$ for each $\epsilon \in\left(0, \epsilon_{1}\right]$ exactly three roots $z_{j}(\epsilon), j=0,1,2$. Moreover, these roots are real and $z_{j}(\epsilon) \rightarrow z_{j}, j=1,2$, and $z_{0}(\epsilon) \rightarrow+\infty$ as $\epsilon \rightarrow 0^{-}$(as in Lemma 11, here $z_{j}$ denote the zeros of $\chi_{+}(z, 0)$ ). Therefore the steady state $\ln p$ of $(9)$ is hyperbolic and the orbit associated with $\phi(t, \epsilon)$ belongs to the stable manifold of $\phi(t)=\ln p$. Thus $y(t):=\phi(t, \epsilon)-\ln p$ and $y^{\prime}(t)$ have at least the exponential rate of decay at $+\infty$. Now, arguing by contradiction, suppose that the function $y(t)=\phi(t, \epsilon)-\ln p$ oscillates slowly around 0. Clearly, $y(t)$ satisfies equation 111 where $A=B=\epsilon^{-1}, C(t)=-\epsilon^{-1} \int_{0}^{1} f^{\prime}(\phi(t, \epsilon) s+(1-s) \ln p) d s$, $C(+\infty)=-\epsilon^{-1} f^{\prime}(\ln p)=\epsilon^{-1} P>0$. But then Lemma 15 guarantees that $y(t)$ has at most the exponential rate of decay at $+\infty$. This implies that there is a complex zero $z_{j}(\epsilon)=\alpha_{j}(\epsilon)+i \beta_{j}(\epsilon), \beta_{j}(\epsilon)>0, \alpha_{j}(\epsilon)<z_{2}(\epsilon)$, of $\chi_{+}(z, \epsilon)$ and $d(\epsilon) \neq 0, \delta>0, \theta \in \mathbb{R}$, such that

$$
\phi(t, \epsilon)-\ln p=d(\epsilon) e^{\alpha_{j}(\epsilon) t} \cos \left(\beta_{j}(\epsilon) t+\theta\right)+O\left(e^{\left(\alpha_{j}(\epsilon)-\delta\right) t}\right), \quad t \rightarrow+\infty .
$$

Now, by Lemma 21 in [42], $\beta_{j}(\epsilon) \geq 2 \pi / \tau$ and therefore $y(t)$ does not oscillate slowly around 0 . The obtained contradiction completes the proof of Corollary 16.

Clearly, Theorem 1 is a direct consequence of Proposition 13 and Corollaries 12 and 16 .

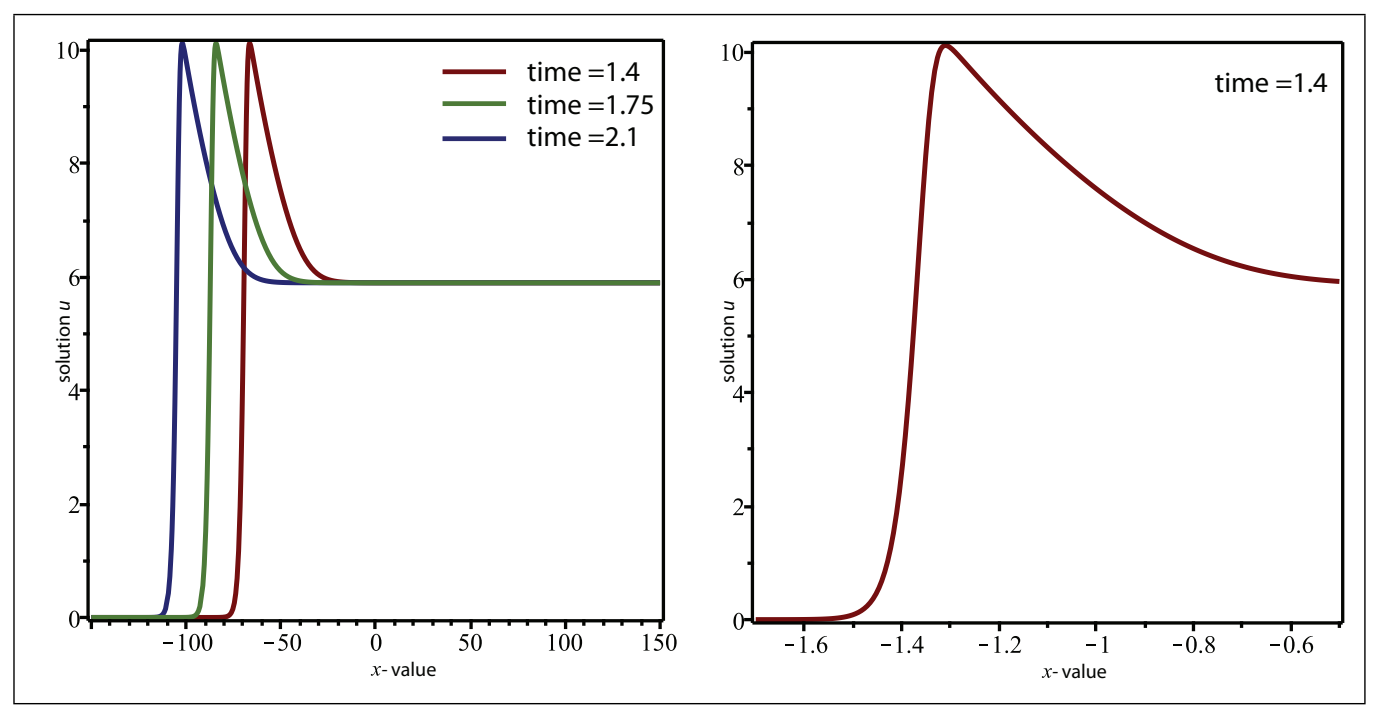

Figure 4: Equation (2) with $\tau=0.07, p=365$ : numerical approximations of the wavefront $u(t, x)=$ $\phi_{c}(x+c t)$ moving leftward with the speed $c \approx 50$ (left); the rescaled profile $\phi_{c}(t c)$ for $c \approx 50$ (right). 
Remark 17. On Fig. 4, we present a fast traveling wave for equation (2) with $\tau=0.07$, $p=365$. The numerical simulations are based on the Crank-Nicholson method which is second-order accurate in both spatial and temporal directions. The initial function is

$$
u_{0}(t, x)= \begin{cases}\exp (0.7 x), & \text { as } x<0, t \in[-\tau, 0] \\ \ln (p), & \text { as } x \geq 0, t \in[-\tau, 0]\end{cases}
$$

The spatial step size is chosen as $\Delta x=0.05$ in the computational domain $x \in[-150,150]$ where the Dirichlet boundary conditions $u(t,-150)=0$ and $u(t, 150)=\ln p$ are imposed. The temporal step size is $\Delta t=0.01$. After some short initial period, the numerical solution $u(t, x)$ behaves like a nm-wave moving leftward with the speed $c \approx 50$. This provides an additional numerical confirmation of the theoretical result in [27] in the case of $n$ m-waves (theoretical speed of propagation is $c=48.26 \ldots$ ). Observe that the rescaled profile $\phi_{c}(c t), c \approx 50$, is rather well approximates the limit profile, $c=\infty$, on the Fig.1.

\subsection{Proof of Theorem 3}

Theorem 3 follows from the following equivalent statement:

Corollary 18. Let $u(t, x)=\phi(x+c t)$ be a wavefront for equation (2). Then the profile $\phi(t)$ is eventually monotone at $+\infty$ if and only if the characteristic function $\chi_{+}(z, \epsilon)=$ $\epsilon z^{2}-z-1-P e^{-z \tau}$ with $\epsilon=c^{-2}$ has at least one negative zero.

Proof. Suppose that equation (2) has an eventually monotone wavefront $u(t, x)=$ $\phi(x+c t)$. Then it follows from the proof of Lemma 25 in 42 that $\chi_{+}(z, \epsilon)$ has a negative zero.

Conversely, suppose that, given a fixed pair $(\tau, c)$ of positive parameters, equation (2) possesses a semi-wavefront $u(t, x)=\phi(x+c t)$ and $\chi_{+}(z, \epsilon)$ has a negative zero $z_{1}<0$. We will denote the latter fact as $(\tau, c) \in \mathcal{D}_{m}$, where

$$
\mathcal{D}_{m}=\left\{\tau \geq 0, c>0 ; z^{2}-c z-1-P e^{-z c \tau}=0 \text { has a negative root }\right\} .
$$

We have to prove that, in such a case, $\phi(t)$ is monotone in some neighborhood of $+\infty$. First, consider $P \in(-1,0]$. Then $\phi(t)$ is necessarily monotone on $\mathbb{R}$ in view of So and Zou main theorem in [37] and the semi-wavefront uniqueness [1, 39, 44]. Second, if $P \in(0,1]$, then the monotonicity of $\phi(t)$ on $\mathbb{R}$ was established in [16, Theorem 2.3].

Hence, in what follows, we can assume that $P>1$. Then the relation (21) in the appendix says that $(\tau, c) \in \mathcal{D}_{m} \subset \mathcal{D}_{s}$. In consequence, since $1-1 / P>\left(P^{2}-P\right) /\left(P^{2}+1\right)$, Proposition 19 guarantees that $\phi(+\infty)=\ln p$ (i.e. $\phi$ is a wavefront). Moreover, $\phi(t)-\ln p$ decays exponentially to 0 as $t \rightarrow+\infty$ (e.g. see [16, Lemma 1.1] or [43, Proposition 5.6]).

Arguing by contradiction, suppose now that $\phi$ oscillates around $\ln p$ on some connected neighborhood $\mathcal{J}$ of $+\infty$. We claim that these oscillations are necessarily slow on J.

Indeed, if $f$ satisfies the feedback condition (18) and $P>1$ (equivalently, $p \in$ $\left(e^{2}, 16.999 \ldots\right)$ or $\ln p \in(2,2.833 \ldots)$, cf. [16, Corollary 2.4]), then the slow oscillation property of $\phi(t)$ follows from Proposition 19 .

Suppose now that $\ln p>2.833$ and consider the oscillating wavefront $\phi(t)$ of equation (9). By Proposition 19, the first three critical points $t_{1}<t_{2}<t_{3}$ of $\phi(t)$ are finite 
and such that $\phi^{\prime}(t)>0$ on $\left(-\infty, t_{1}\right) \cup\left(t_{2}, t_{3}\right), \phi^{\prime}(t)<0$ on $\left(t_{1}, t_{2}\right)$ and $\phi\left(t_{1}\right)>\ln p$, $\phi\left(t_{2}\right)<\ln p<\phi\left(t_{2}-\tau\right)$. We will prove that $\phi(t)>1$ for all $t \geq t_{1}$. First, we observe that, by [43, Lemma 4.2]

$$
\phi\left(t_{2}\right)=\xi\left(t_{2}-s_{2}\right)\left\{\ln p+\frac{1}{\epsilon(\nu-\lambda)} \int_{s_{2}}^{t_{2}}\left(e^{\lambda\left(s_{2}-u\right)}-e^{\nu\left(s_{2}-u\right)}\right) f(\phi(u-\tau)) d u\right\},
$$

where $\lambda<0<\nu$ denote the roots of the equation $\epsilon z^{2}-z-1=0, s_{2}$ is the unique point in $\left(t_{1}, t_{2}\right)$ where $\phi\left(s_{2}\right)=\ln p$ and $\xi(x)=(\nu-\lambda) /\left(\nu e^{-\lambda x}-\lambda e^{-\nu x}\right)$. Since $s_{2} \in\left(t_{2}-\tau, t_{2}\right)$, $(\tau, c) \in \mathcal{D}_{m} \subset \mathcal{D}_{s}, P>1.833$ and $\xi^{\prime}(x)<0$ for $x>0$, we immediately find that

$$
\phi\left(t_{2}\right)>\xi(\tau) \ln p>\left(1-\frac{1}{P}\right)(P+1)=P-\frac{1}{P} \geq 1.833-1 / 1.833>1 .
$$

Thus $\phi(t)>1$ for all $t \in\left[t_{1}, t_{3}\right)$ and if $\phi(s) \leq 1$ at some point $s \geq t_{3}$, then there exists the leftmost critical point $t_{*}>t_{3}$ such that $\phi\left(t_{*}\right)=\min \left\{\phi(s), s \in\left[t_{1}, t_{*}\right]\right\} \leq 1, \phi^{\prime}\left(t_{*}\right)=0$ and $\phi^{\prime \prime}\left(t_{*}\right) \geq 0$. In particular, $\phi\left(t_{*}-\tau\right)>\phi\left(t_{*}\right)$. Since, in addition, equation (9) implies that $\phi\left(t_{*}\right) \geq f\left(\phi\left(t_{*}-\tau\right)\right)$, we obtain that $\phi\left(t_{*}-\tau\right)>\ln p$. Therefore $\phi\left(s_{*}\right)=\ln p$ for some $s_{*} \in\left(t_{*}-\tau, t_{*}\right)$. Invoking again formula $[16)$, we find that $\phi\left(t_{*}\right)>\xi(\tau) \ln p>1$. This contradiction proves that actually $\phi(t)>1$ for all $t \geq t_{1}$. Therefore the feedback condition $(18)$ is satisfied on the set $\left\{\phi(t), t \geq t_{1}\right\} \subset[1,+\infty)$. Since the slow oscillation property of $\phi(t)$ follows now from Proposition 19 and Corollary 14 in [42, the claim is proved.

Finally, arguing as in the second half of the proof of Corollary 16, we conclude that the assumption $(\tau, c) \in \mathcal{D}_{m}$ is not compatible with the existence of slowly oscillating (around $\ln p$ ) and exponentially converging (toward $\ln p$ ) wavefront $\phi(t)$. Therefore $\phi(t)$ is monotone at $+\infty$.

\section{Appendix}

In this section, we are use the notation

$$
\lambda=\frac{c\left(c-\sqrt{c^{2}+4}\right)}{2}, \quad \nu=\frac{c\left(c+\sqrt{c^{2}+4}\right)}{2}, \quad P=\ln p-1, \quad \epsilon=c^{-2} .
$$

The next assertion follows from [43, Theorem 1.1] and [42, Theorems 3, 13], is instrumental in proving Theorem 3 (or Corollary 18) of this paper and is given for the convenience of the reader. We consider the following restrictions on the nonlinearity $f$ :

(H) Let $f \in C^{3}\left(\mathbb{R}_{+}, \mathbb{R}_{+}\right)$have only one critical point $x_{M}$ (maximum) and assume that $f(0)=0$ and $f^{\prime}(0)>1$. Suppose further that $0<f(x) \leq f^{\prime}(0) x, x>0$, that the equation $f(x)=x$ has exactly two roots $0, \kappa>0$ with $\Gamma:=f^{\prime}(\kappa)<0$, and that the Schwarz derivative $S f$ is negative for all $x>0, x \neq x_{M}$ :

$$
(S f)(x)=f^{\prime \prime \prime}(x)\left(f^{\prime}(x)\right)^{-1}-(3 / 2)\left(f^{\prime \prime}(x)\left(f^{\prime}(x)\right)^{-1}\right)^{2}<0 .
$$


Proposition 19. Assume $\mathbf{( H )}$, suppose that the equation $\epsilon z^{2}-z-1+f^{\prime}(0) e^{-z \tau}=0$ has at least one positive root, and

$$
\frac{\nu-\lambda}{\nu e^{-\lambda \tau}-\lambda e^{-\nu \tau}} \geq \frac{\Gamma^{2}+\Gamma}{\Gamma^{2}+1}
$$

Then equation (9) has a positive wavefront $\phi(t)$. Furthermore, if $\phi(t)$ is non-monotone on $\mathbb{R}$, then there exist $t_{3} \geq t_{2}>t_{1}, t_{1} \in \mathbb{R}, t_{2}, t_{3} \in \mathbb{R} \cup\{+\infty\}$, such that $\phi^{\prime}(t)>0$ on $\left(-\infty, t_{1}\right) \cup\left(t_{2}, t_{3}\right), \phi\left(t_{1}\right)>\kappa$, and $\phi^{\prime}(t)<0$ on $\left(t_{1}, t_{2}\right)$. If $t_{2}$ is finite then $\phi\left(t_{2}\right)<\kappa<$ $\phi\left(t_{2}-\tau\right)$ and $t_{3}>t_{2}$. Finally, if $f$ satisfies the feedback condition

$$
(f(x)-\kappa)(x-\kappa)<0, x \in\left(f\left(f\left(x_{M}\right)\right), f\left(x_{M}\right)\right) \backslash\{\kappa\},
$$

then $\phi(t)$ is either eventually monotone or oscillates slowly (see Definition 14) around $\kappa$.

Lemma 20. For each fixed $c>0$ and $P>1$, equation

$$
\Phi(\tau, c):=\frac{\nu-\lambda}{\nu e^{-\lambda \tau}-\lambda e^{-\nu \tau}}=1-\frac{1}{P}
$$

has a unique positive root $\tau=\tau(c)$. The function $\tau(c):(0,+\infty) \rightarrow(0,+\infty)$ is smooth and has a finite limit $\tau(+\infty)=\ln (P /(P-1))=: \hat{\tau}>0$.

Proof. It is easy to check that $\partial \Phi(\tau, c) / \partial \tau<0$ for all $\tau>0, c>0$. Taking into account that $\Phi(0, c)=1, \Phi(+\infty, c)=0$ and $P>1$, we deduce the existence of the unique solution $\tau=\tau(c)$ of equation (19). Now, let $\hat{\tau}$ be the limit of sequence $\tau\left(c_{j}\right)$ with $c_{j} \rightarrow+\infty$. Then necessarily $e^{-\hat{\tau}}=1-1 / P$ which proves the uniqueness and finiteness of $\hat{\tau}>0$. Thus $\tau(+\infty)=\ln (P /(P-1))$.

In the first quadrant of the plane $(\tau, c)$, given a fixed number $P>1$, together with the above defined set $\mathcal{D}_{m}$ of parameters $(\tau, c)$, we consider also the following domain

$$
\mathcal{D}_{s}=\left\{\tau \geq 0, c>0, \Phi(\tau, c)=\frac{\nu-\lambda}{\nu e^{-\lambda \tau}-\lambda e^{-\nu \tau}} \geq 1-\frac{1}{P}\right\} .
$$

Lemma 20 shows that $\mathcal{D}_{s}=\left\{(\tau, c) \in \mathbb{R}_{+}^{2}: 0 \leq \tau \leq \tau(c), c>0\right\}$. On the other hand, it was established in [16, Section 2.3] that $\mathcal{D}_{m}=\left\{(\tau, c) \in \mathbb{R}_{+}^{2}: 0 \leq \tau \leq T(c), c>0\right\}$, where $T(c)$ is defined as the unique positive solution of equation

$$
\frac{e c^{2} \tau^{2}}{2+\sqrt{c^{4} \tau^{2}+4 c^{2} \tau^{2}+4}} \exp \left(\frac{\sqrt{c^{4} \tau^{2}+4 c^{2} \tau^{2}+4}-c^{2} \tau}{2}\right)=\frac{1}{P}
$$

By [16, Lemma 1.1], $T(c)$ is a smooth decreasing function such that $T\left(0^{+}\right)=+\infty$, $T(+\infty)=T_{*}$, where $P e T_{*} e^{T_{*}}=1$. Observe that $P e \hat{\tau} e^{\hat{\tau}}=e P^{2}(P-1)^{-1} \ln (P /(P-1))>1$ for $P>1$ so that $\tau(+\infty)=\hat{\tau}>T_{*}=T(+\infty)$. In fact, the next lemma assures that $T(c)<\tau(c)$ for all $c>0$ so that

$$
\mathcal{D}_{m} \subset \mathcal{D}_{s}
$$


Lemma 21. For each $\tau>0$ and $c>0$, it holds that

$$
\frac{e c^{2} \tau^{2}}{2+\sqrt{c^{4} \tau^{2}+4 c^{2} \tau^{2}+4}} \exp \left(\frac{\sqrt{c^{4} \tau^{2}+4 c^{2} \tau^{2}+4}-c^{2} \tau}{2}\right)>1-\frac{\nu-\lambda}{\nu e^{-\lambda \tau}-\lambda e^{-\nu \tau}} .
$$

Proof. With $h=c \tau$, inequality (22) is equivalent to

$$
l=e h^{2} \exp \left(\frac{2\left(h^{2}+1\right)}{\sqrt{h^{2}\left(c^{2}+4\right)+4}+c h}\right)>\left(2+\sqrt{h^{2}\left(c^{2}+4\right)+4}\right)\left(1-\frac{\nu-\lambda}{\nu e^{-\lambda \tau}-\lambda e^{-\nu \tau}}\right)=r .
$$

For fixed $h>0$, we equation maximize $r$ and minimize $l$ with respect to $c \in \mathbb{R}_{+}$. The second task is easy: $\mathfrak{l}=\min _{c \geq 0} l=e h^{2}$. To maximize $r$, we will introduce the new variable $z=\left(c+\sqrt{c^{2}+4}\right) / 2, z \in[1,+\infty)$. Then $r$ takes the form

$$
r=r(z):=\left(2+\sqrt{h^{2}\left(z+\frac{1}{z}\right)^{2}+4}\right)\left(1-\frac{z^{2}+1}{z^{2} e^{h / z}+e^{-h z}}\right) .
$$

We will further simplify $r$ :

$$
r(z)<r_{1}(z):=\left(4+h\left(z+\frac{1}{z}\right)\right)\left(1-\frac{z^{2}+1}{z^{2} e^{h / z}+e^{-h z}}\right) .
$$

We claim that, for all $z \geq 1, r_{1}(z) \leq e h^{2}$. First, we rewrite this last inequality in the following equivalents forms:

$$
\begin{gathered}
1 \leq \frac{t^{2}+1}{e^{t h}+t^{2} e^{-h / t}}+\frac{e h^{2}}{4+h\left(t+\frac{1}{t}\right)}, \quad t=1 / z \in(0,1], h>0, \\
1 \leq \frac{t^{2}+1}{e^{M t^{2}}+t^{2} e^{-\sigma}}+\frac{e \sigma^{2} t^{2}}{4+\sigma\left(t^{2}+1\right)}, \quad t \in(0,1], \sigma=\frac{h}{t}>0, \\
1 \leq \frac{w e^{\sigma}}{e^{w \sigma}+w-1}+\frac{e \sigma^{2}(w-1)}{4+w \sigma}, \quad w=t^{2}+1 \in(1,2], \sigma>0, \\
\quad \frac{e^{w \sigma}+w-1-w e^{\sigma}}{e^{w \sigma}+w-1} \leq \frac{e \sigma^{2}(w-1)}{4+w \sigma}, \quad w \in(1,2], \sigma>0, \\
\quad\left(e^{w \sigma}+w-1-w e^{\sigma}\right)(4+w \sigma) \leq e \sigma^{2}(w-1)\left(e^{w \sigma}+w-1\right), \\
A(w, \sigma):=e^{w \sigma}\left(e \sigma^{2}(w-1)-(4+w \sigma)\right)+e \sigma^{2}(w-1)^{2}+(4+w \sigma)\left(1+w\left(e^{\sigma}-1\right)\right) \geq 0,
\end{gathered}
$$

to be proved for all $w \in(1,2], \sigma>0$. It is convenient to represent $A(w, \sigma)$ in the form of the power series:

$$
\begin{gathered}
A(w, \sigma)=w \sum_{k=2}^{+\infty} \frac{A_{k}(w)}{k !} \sigma^{k}=w \sigma^{2}\left(\frac{A_{2}(w)}{2 !}+\frac{A_{3}(w)}{3 !} \sigma+\frac{A_{4}(w)}{4 !} \sigma^{2}\right)+w \sum_{k=5}^{+\infty} \frac{A_{k}(w)}{k !} \sigma^{k}= \\
\frac{w \sigma^{2}}{4 !}\left(12 A_{2}(w)+4 A_{3}(w) \sigma+A_{4}(w) \sigma^{2}\right)+w \sum_{k=5}^{+\infty} \frac{A_{k}(w)}{k !} \sigma^{k}=: B(w, \sigma)+C(w, \sigma),
\end{gathered}
$$


where $A_{2}(w)=2(e-2)(w-1)>0, w \in(1,2]$,

$$
\begin{gathered}
A_{3}(w)=(w-1)(6 e-7 w-4)>0, \quad w \in(1,1.75], \\
A_{4}(w)=-8 w^{3}+12 e w^{2}-12 e w+4 w+4=-4(w-1)\left(2 w^{2}+(2-3 e) w+1\right)>0, \quad w \in(1,2], \\
A_{k}(w)=-w^{k-1}(k+4)+e k(k-1) w^{k-2}-e k(k-1) w^{k-3}+4+k w, \quad k \geq 3 .
\end{gathered}
$$

It follows from the above expressions that $A_{k}(1)=0$ for all $k \geq 2$ and since for all $k \geq 5$, it holds that

$$
A_{k}^{\prime}(1)=-(k-1)(k+4)+e k(k-1)(k-2)-e k(k-1)(k-3)+k=(k-1)(e k-k-4)+k>0,
$$

as well as for $w \in(1,2]$

$$
\begin{gathered}
A_{k}^{\prime \prime}(w)=-w^{k-3}(k+4)(k-1)(k-2)+e k(k-1)(k-2)(k-3) w^{k-4}-e k(k-1)(k-3)(k-4) w^{k-5} \\
=w^{k-5}(k-1)\left\{-w^{2}(k+4)(k-2)+e k(k-2)(k-3) w-e k(k-3)(k-4)\right\}>0,
\end{gathered}
$$

we conclude that $A_{k}(w)>0$ for all $w \in(1,2], k=2,4,5,6, \ldots$ and $A_{3}(w)>0$ for all $w \in(1,1.75]$. This proves that $A(w, \sigma)>0$ for all $w \in(1,1.75], \sigma>0$, and $C(w, \sigma)>0$ for all $w \in(1,2], \sigma>0$. Hence, to complete the proof of the positivity of $A(w, \sigma)$ for all $\sigma>0$ and $w \in(1,2]$, it suffices to establish that $B(w, \sigma)>0$ for all $w \in[1.75,2], \sigma>0$. Now, $B(w, \sigma)$ or, equivalently, $12 A_{2}(w)+4 A_{3}(w) \sigma+A_{4}(w) \sigma^{2}$ will be positive for all $\sigma>0$ and for some fixed $w \in(1,2]$ if the discriminant

$$
\begin{gathered}
D(w)=16\left(A_{3}^{2}(w)-3 A_{2}(w) A_{4}(w)\right)= \\
16(w-1)^{2}\left((6 e-7 w-4)^{2}+24(e-2)\left(2 w^{2}+(2-3 e) w+1\right)\right)
\end{gathered}
$$

will be negative for this value of $w$. Observe that $D(w)$ is the product of two quadratic polynomials so that it is immediate to see that $D(w)<0$ for all $w \in(1,2]$. In a consequence, $B(w, \sigma)>0$ for all $w \in(1,2]$ and $\sigma>0$ and also $A(w, \sigma)>0$.

Finally, the positivity of $A(w, \sigma)$ assures that $r=r(z)<r_{1}(z) \leq e h^{2}=\mathfrak{l} \leq l$.

\section{Acknowledgments}

The work of Karel Hasík, Jana Kopfová and Petra Nábělková was supported by the institutional support for the development of research organizations IČO 47813059. This work was realized during a stay of Sergei Trofimchuk at the Silesian University in Opava, Czech Republic. This stay was possible due to the support of the Silesian University in Opava and the European Union through the project CZ.02.2.69/0.0/0.0/16_027/0008521. S. Trofimchuk was also partially supported by FONDECYT (Chile), project 1190712. 


\section{References}

[1] M. Aguerrea, C. Gomez, S. Trofimchuk, On uniqueness of semi-wavefronts (Diekmann-Kaper theory of a nonlinear convolution equation re-visited), Math. Ann. 354 (2012) 73-109.

[2] O. Arino, A note on "The discrete Lyapunov function...," J. Differential Equations 104 (1993), $169-181$.

[3] M. Bani-Yaghoub, G.-M. Yao, M. Fujiwara, D.E. Amundsen, Understanding the interplay between density dependent birth function and maturation time delay using a reaction-diffusion population model, Ecological Complexity 21 (2015) 14-26.

[4] L. Berezansky, E. Braverman, L. Idels, Nicholson's blowflies differential equations revisited: Main results and open problems, Appl. Math. Modelling 34 (2010) 1405-1417.

[5] Y. M. Berezansky, Z. G. Sheftel, G. F. Us, Functional Analysis, Vol. I-Birkhäuser Basel, 1996.

[6] Y. Cao, The discrete Lyapunov function for scalar differential delay equations, J. Differential Equations 87 (1990), 365-390.

[7] I.-L. Chern, M. Mei, X.-F. Yang, Q.-F. Zhang, Stability of non-monotone critical traveling waves for reaction-diffusion equations with time-delay, J. Differential Equations 259 (2015) 1503-1541.

[8] A. Ducrot, G. Nadin, Asymptotic behaviour of traveling waves for the delayed Fisher-KPP equation, J. Differential Equations 256 (2014) 3115-3140.

[9] G.P. Egorychev, Integral representation and the computation of combinatorial sums, American Mathematical Society, 1984.

[10] A. Ivanov, C. Gomez, S. Trofimchuk. On the existence of non-monotone non-oscillating wavefronts, J. Math. Anal. Appl. 419 (2014) 606-616.

[11] J. Fang, X.-Q. Zhao, Monotone wavefronts of the nonlocal Fisher-KPP equation, Nonlinearity 24 (2011) 3043-3054 .

[12] T. Faria, W. Huang, J. Wu, Traveling waves for delayed reaction-diffusion equations with non-local response, Proc. R. Soc. A 462 (2006) 229-261.

[13] T. Faria, S. Trofimchuk, Non-monotone travelling waves in a single species reaction-diffusion equation with delay, J. Differential Equations 228 (2006) 357-376.

[14] T. Faria, S. Trofimchuk, Positive traveling fronts for reaction-diffusion systems with distributed delay, Nonlinearity 23 (2010) 2457-2481.

[15] T. Faria, S. Trofimchuk, Positive heteroclinics and traveling waves for scalar population models with a single delay, Appl. Math. Comput. 185 (2007) 594-603.

[16] A. Gomez, S. Trofimchuk, Global continuation of monotone wavefronts, J. London Math. Soc. 89 (2014) 47-68.

[17] S.A. Gourley, Travelling fronts in the diffusive Nicholson's blowflies equation with distributed delays, Mathematical and Computer Modelling 32 (2000) 843-853.

[18] S. A. Gourley and S. Ruan, Dynamics of the diffusive Nicholson's blowflies equation with distributed delay, Proc. R. Soc. Edinb. Sect. A 130 (2000) 1275-1291.

[19] S.A. Gourley, J. W.-H. So and J. Wu, Non-locality of reaction-diffusion equations induced by delay: biological modeling and nonlinear dynamics, J. Math. Sci. 124 (2004) 5119-5153.

[20] S. Guo, J. Zimmer, Stability of traveling wavefronts in discrete reaction-diffusion equations with nonlocal delay effects, Nonlinearity 28 (2015) 463-492.

[21] W. S. C. Gurney, S. P. Blythe, and R. M. Nisbet. Nicholson's blowflies revisited, Nature, 287:17-21, 1980.

[22] K. Hasík, J. Kopfová, P. Nábělková, S. Trofimchuk, On the geometric diversity of wavefronts for the scalar Kolmogorov ecological equation, e-print arXiv:1903.10339v1

[23] M. Lewis, B. Li, H. Weinberger, Spreading speed and linear determinacy for two-species competition models, J. Math. Biol. 45 (2002) 219-233.

[24] W.T. Li, S.G. Ruan, Z.C. Wang, On the diffusive Nicholson's blowflies equation with nonlocal delays, J. Nonlinear Sci. 17 (2007) 505-525.

[25] D. Liang, J. Wu, Travelling waves and numerical approximations in a reaction advection diffusion equation with nonlocal delayed effects, J. Nonlinear Sci. 13 (2003) 289-310.

[26] C.-K. Lin, M. Mei, On travelling wavefronts of the Nicholson's blowflies equations with diffusion, Proc. R. Soc. Edinb. A 140 (2010) 135-152.

[27] C.-K. Lin, C.-T. Lin, Y. Lin, M. Mei, Exponential stability of nonmonotone traveling waves for Nicholson's blowflies equation, SIAM J. Math. Anal. 46 (2014) 1053-1084.

[28] G. Lin, Travelling waves in the Nicholson's blowflies equation with spatio-temporal delay. Appl. Math. Comput. 209(2), 314-326 (2009) 
[29] E. Liz, V. Tkachenko and S. Trofimchuk, A global stability criterion for scalar functional differential equations, SIAM J. Math. Anal. 35 (2003) 596-622.

[30] J. Mallet-Paret, Morse decompositions for differential delay equations, J. Differential Equations 72 (1988), 270-315.

[31] J. Mallet-Paret, G. Sell, Systems of delay differential equations I: Floquet multipliers and discrete Lyapunov functions, J. Differential Equations 125 (1996) 385-440.

[32] A. N. Murovtsev, Global analyticity of solutions of nonlinear functional-differential equations representable by Dirichlet series, Ukrainian Math. J., 58 (2006) 1448-1457.

[33] J. D. Murray, Mathematical Biology, Springer, Berlin, 1989.

[34] R. D. Nussbaum, Periodic solutions of analytic functional differential equations are analytic, Michigan Math. J., 20 (1973) 249-255.

[35] H.L. Smith, Monotone dynamical systems, Amer. Math. Soc., Providence, 1995.

[36] J. W.-H. So, J. Wu, X. Zou, A reaction-diffusion model for a single species with age structure. I Travelling wavefronts on unbounded domains, Proc. Roy. Soc. London. Ser. A, 457 (2001) 18411853.

[37] J. So, X. Zou, Traveling waves for the diffusive Nicholson's blowflies equation, Appl. Math. Comput. 122 (2001) 385-392.

[38] A. Solar, S. Trofimchuk, Speed selection and stability of wavefronts for delayed monostable reactiondiffusion equations. J. Dynam. Differential Equations 28 (2016) 1265-1292.

[39] H.R. Thieme, X.-Q. Zhao Asymptotic speeds of spread and traveling waves for integral equations and delayed reaction-diffusion models J. Differential Equations, 195 (2003) 430-470

[40] E. Trofimchuk, M. Pinto, S. Trofimchuk, Existence and uniqueness of monotone wavefronts in a nonlocal resource-limited model, Proc. R. Soc. Edinb. Sect. A (2019), doi:10.1017/prm.2019.31.

[41] E. Trofimchuk, M. Pinto and S. Trofimchuk. Monotone waves for non-monotone and non-local monostable reaction-diffusion equations, J. Differential Equations 261 (2016), 203-1236.

[42] E. Trofimchuk, V. Tkachenko, S. Trofimchuk, Slowly oscillating wave solutions of a single species reaction-diffusion equation with delay, J. Differential Equations, 245 (2008) 2307-2332.

[43] E. Trofimchuk, S. Trofimchuk, Admissible wavefront speeds for a single species reaction-diffusion equation with delay, Discrete Contin. Dyn. Syst. A 20 (2008), 407-423.

[44] S.-L. Wu, S.-Y. Liu, Uniqueness of non-monotone traveling waves for delayed reaction-diffusion equations, Applied Mathematics Letters, 22 (2009) 1056-1061.

[45] J.W.-H. So, Y. Yang, Dirichlet problem for the diffusive Nicholson's blowflies equation, J. Differential Equations, 150 (1998) 317-348.

[46] T. Yi, Y. Chen, J. Wu, Unimodal dynamical systems: Comparison principles, spreading speeds and travelling waves, J. Differential Equations, 254 (2013) 3538-3572.

[47] C. Zhang, X. Yan, Wavefront solutions in diffusive Nicholson's blowflies equation with nonlocal delay, Appl. Math. Mech. 31 (2010) 385-392. 